\title{
Monte Carlo Simulation of Background and Source Measurements with CSG and CAD Geometries
}

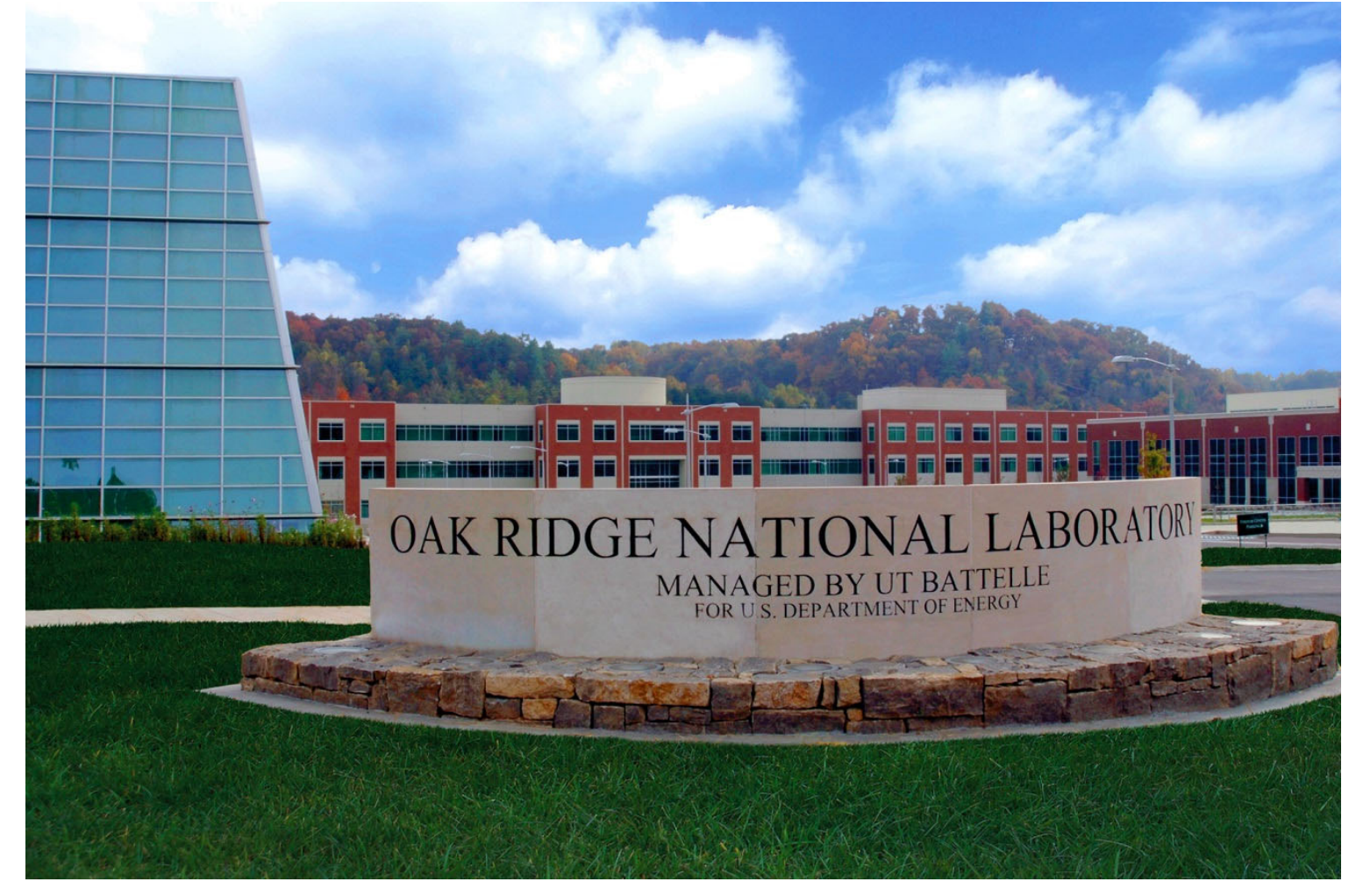

Douglas E. Peplow Gregory G. Davidson Cihangir Celik Elliott D. Biondo Alexandra C. Hackett William R. Ray Daniel E. Archer James M. Ghawaly, Jr.
Andrew D. Nicholson Michael J. Willis Brian J. Quiter Mark S. Bandstra Ross E. Meyer Chun Ho Chow Ian R. Stewart Jeffrey O. Johnson

June 2021 


\title{
DOCUMENT AVAILABILITY
}

Reports produced after January 1, 1996, are generally available free via US Department of Energy (DOE) SciTech Connect.

Website www.osti.gov

Reports produced before January 1, 1996, may be purchased by members of the public from the following source:

\author{
National Technical Information Service \\ 5285 Port Royal Road \\ Springfield, VA 22161 \\ Telephone 703-605-6000 (1-800-553-6847) \\ TDD 703-487-4639 \\ Fax 703-605-6900 \\ E-mail info@ntis.gov \\ Website http://classic.ntis.gov/
}

Reports are available to DOE employees, DOE contractors, Energy Technology Data Exchange representatives, and International Nuclear Information System representatives from the following source:

Office of Scientific and Technical Information

PO Box 62

Oak Ridge, TN 37831

Telephone 865-576-8401

Fax 865-576-5728

E-mail reports@osti.gov

Website https://www.osti.gov/

This report was prepared as an account of work sponsored by an agency of the United States Government. Neither the United States Government nor any agency thereof, nor any of their employees, makes any warranty, express or implied, or assumes any legal liability or responsibility for the accuracy, completeness, or usefulness of any information, apparatus, product, or process disclosed, or represents that its use would not infringe privately owned rights. Reference herein to any specific commercial product, process, or service by trade name, trademark, manufacturer, or otherwise, does not necessarily constitute or imply its endorsement, recommendation, or favoring by the United States Government or any agency thereof. The views and opinions of authors expressed herein do not necessarily state or reflect those of the United States Government or any agency thereof. 
Nuclear Energy and Fuel Cycle Division

\section{MONTE CARLO SIMULATION OF BACKGROUND AND SOURCE MEASUREMENTS WITH CSG AND CAD GEOMETRIES}

\author{
Douglas E. Peplow* \\ Gregory G. Davidson* \\ Cihangir Celik* \\ Elliott D. Biondo* \\ Alexandra C. Hackett* \\ William R. Ray* \\ Daniel E. Archer* \\ James M. Ghawaly, Jr.*
}

\author{
Andrew D. Nicholson* \\ Michael J. Willis* \\ Brian J. Quiter $\dagger$ \\ Mark S. Bandstra $\uparrow$ \\ Ross E. Meyer $\dagger$ \\ Chun Ho Chow $\dagger$ \\ Ian R. Stewart: \\ Jeffrey O. Johnson*
}

* Oak Ridge National Laboratory

$\uparrow$ Lawrence Berkeley National Laboratory

$\$$ University of Tennessee

June 2021

Prepared by

OAK RIDGE NATIONAL LABORATORY

Oak Ridge, TN 37831-6283

managed by

UT-BATTELLE LLC

for the

US DEPARTMENT OF ENERGY

under contract DE-AC05-00OR22725 



\section{CONTENTS}

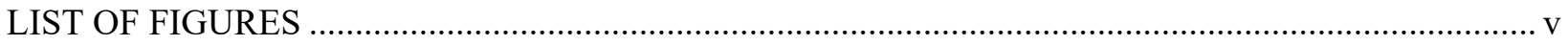

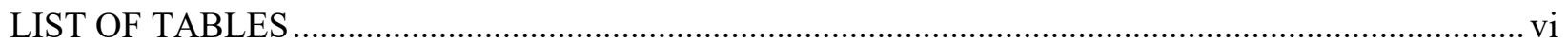

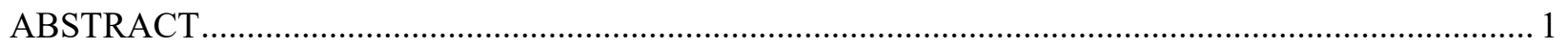

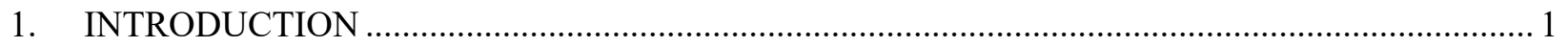

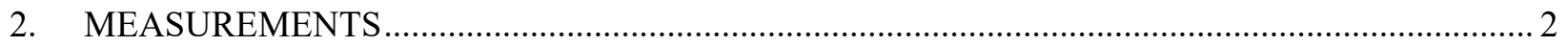

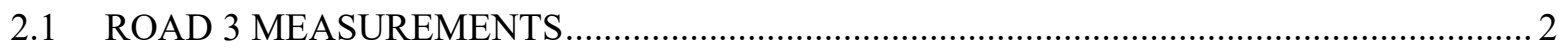

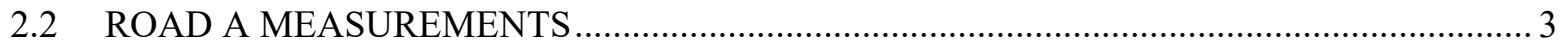

2.3 MEASUREMENTS FOR BACKGROUND K, U, AND TH CONCENTRATIONS ............... 3

2.4 MEASUREMENTS USING A MOBILE RADIATION DETECTION SYSTEM ................. 7

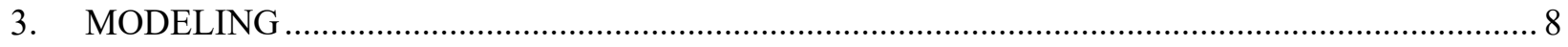

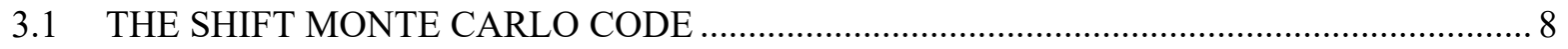

3.1.1 Hybrid Monte Carlo/Deterministic Capabilities in Shift ............................................. 8

3.1.2 Computing the Background Radiation Source using Shift ....................................... 9

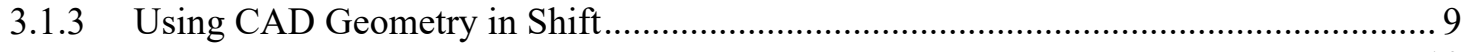

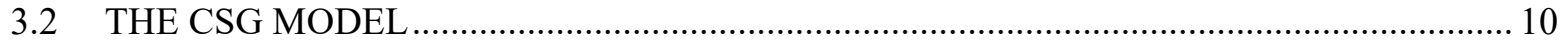

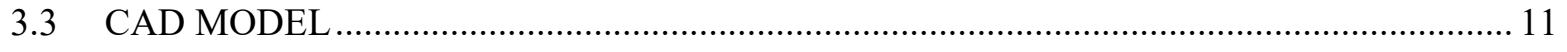

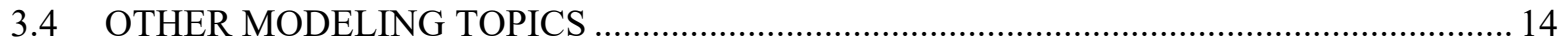

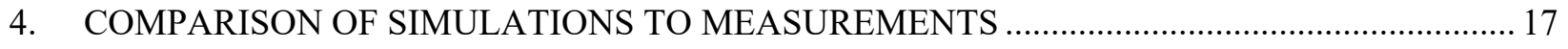

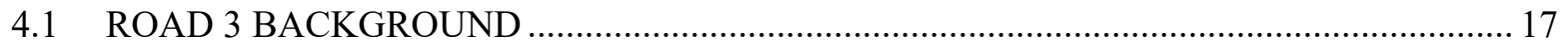

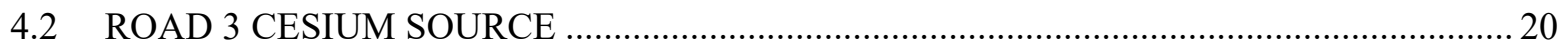

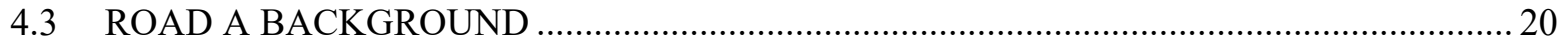

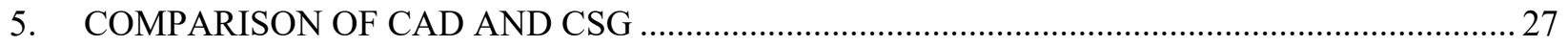

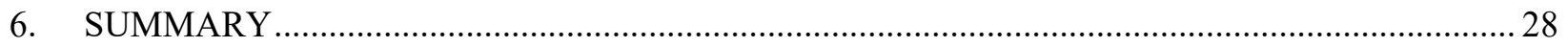

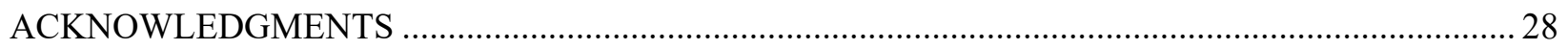

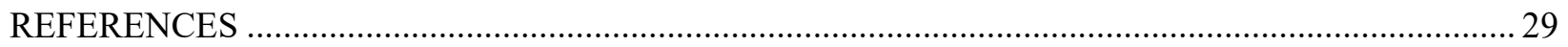




\section{LIST OF FIGURES}

Figure 1. The coordinate system used for detector positions at the FTIG CACTF has its origin at the intersection of the centerlines of Road 3 and Road B.

Figure 2. Overhead view of the main area of the FTIG CACTF with grass (green), asphalt roads (gray), sidewalks (light gray), gravel areas (light yellow), concrete (white), and buildings (red, blue, tan, dark gray, and brown).

Figure 3. Experimental measurement setup based on an RSI detector used by RSL-Andrews................... 5

Figure 4. Measurements along Road 3 taken during the OPTUS-1 campaign used RSI $2 \times 4 \times 16$ in. $\mathrm{NaI}$ detectors resting on a table....

Figure 5. Detector in a cart with associated equipment, used by ORNL (Image courtesy of ORNL).

Figure 6. Locations of background measurements taken with the four ORNL carts.

Figure 7. The CSG model of the FTIG viewed from the northeast. Included are asphalt roads (gray), concrete intersections and roads (white), gravel areas (light yellow) and nine buildings (light gray, red, blue, tans).

Figure 8. Top-down view of the semantically labeled point cloud produced by RadMAP during $165 \mathrm{~s}$ of driving around the FTIG facility.

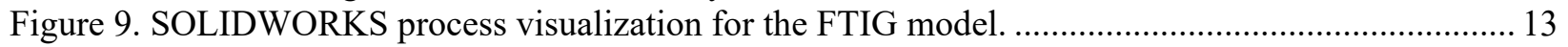

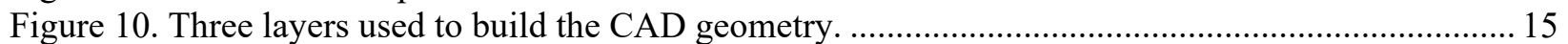

Figure 11. The FTIG CAD model with all layers merged and materials assigned.................................. 16

Figure 12. Road 3 background count rate spectra at location $\mathrm{x}=10 \mathrm{~m}$ with measurement data and both CSG and CAD simulations.

Figure 13. Road 3 background spectra at location $\mathrm{x}=10 \mathrm{~m}$ for the low-energy portion of the spectra (left) and near the ${ }^{40} \mathrm{~K}$ peak (right), with measurement data and both CSG and CAD simulations.

Figure 14. Road 3 background count rate spectra at location $\mathrm{x}=40 \mathrm{~m}$ with measurement data and both CSG and CAD simulations.

Figure 15. Road 3 background spectra at location $\mathrm{x}=40 \mathrm{~m}$ for the low-energy portion of the spectra (left) and near the ${ }^{40} \mathrm{~K}$ peak (right), with measurement data and both CSG and CAD simulations.

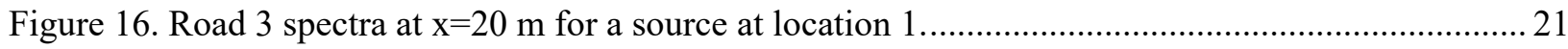

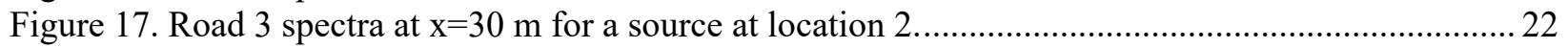

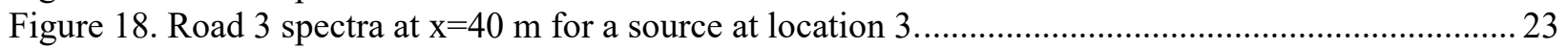

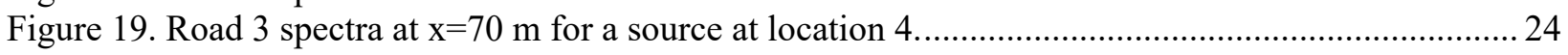

Figure 20. Road A background count rate spectra at location 1 with measurement data and both

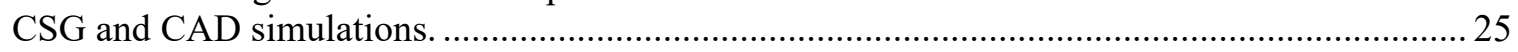

Figure 21. Road A background spectra at location 1 for the low-energy portion of the spectra (left) and near the ${ }^{40} \mathrm{~K}$ peak (right), with measurement data and both CSG and CAD simulations.

Figure 22. Road A background count rate spectra at location 6 with measurement data and both

CSG and CAD simulations.

Figure 23. Road A background spectra at location 1 for the low-energy portion of the spectra (left) and near the ${ }^{40} \mathrm{~K}$ peak (right), with measurement data and both $\mathrm{CSG}$ and CAD simulations. 


\section{LIST OF TABLES}

Table 1. Detector and source locations for the Road 3 source measurements....................................... 5

Table 2. Particle processing rate for the twelve simulations (millions of particles per minute) ................ 27 


\begin{abstract}
It is difficult to detect radioactive sources in an urban environment due to the large magnitude and variability of background radiation. To support the search mission of the National Nuclear Security Administration, a project was undertaken to determine if first-principles modeling and simulation could be used to accurately predict the response of radiation detectors in urban environments. This study demonstrated that the simulated detector responses to photon radiation from an isotopic source and from the background compare well to benchmark-quality measurements in a large urban-like test environment. Simulation results using a traditional combinatorial solid geometry and a computer-aided design (CAD) model generated from light detection and ranging (LiDAR) measurements gave very similar detector responses. With this validation, further simulations were used to predict detector responses for various situations in real urban settings.
\end{abstract}

\title{
1. INTRODUCTION
}

The purpose of the Modeling Urban Scenarios and Experiments (MUSE) project [1] is to demonstrate the ability to use modeling and simulation to advance the state of the art in missions related to the search for nuclear materials in urban environments. The project is a joint effort between Oak Ridge National Laboratory (ORNL) and Lawrence Berkeley National Laboratory (LBNL). In the earlier measurement phases [2], the Remote Sensing Laboratory - Andrews (RSL-A) was also a MUSE collaborator. The overall goal is to create a virtual testbed to quickly and accurately predict detector responses to background and threat sources using detailed first-principles radiation transport simulations. By combining modern Monte Carlo radiation transport tools with advanced variance reduction and computer aided design (CAD) geometries derived from unstructured point clouds, detailed simulations of search scenarios or experiments can be constructed and completed in reasonable amounts of time. The main problem of interest to the MUSE project is the wide-area exterior search using a vehicle-mounted detector to look for a threat source somewhere in a city, but not inside a building (the shielding provided by large buildings is too great). Threat source detection and identification depend on differentiating a signal from background. The real difficulty in urban environments is the large amount of background radiation, which is mainly due to the naturally occurring radioactive materials (NORM) from the roadways, sidewalks, curbs, and exterior materials of buildings.

When modeling urban environments, it is essential to model the background accurately. Sandness et al. [3] show that the background measured in the desert near Pacific Northwest National Laboratory (PNNL) consists of gamma rays almost entirely from ${ }^{40} \mathrm{~K},{ }^{238} \mathrm{U} /{ }^{235} \mathrm{U}$ and their daughters, and ${ }^{232} \mathrm{Th}$ and its daughters present in the soil and rock. Some ${ }^{137} \mathrm{Cs}$ in the soil is also noted. Background measurements taken in a boat on a lake, with a large thickness of water shielding the rock/soil below, showed a cosmic ray component, as well as peaks from radon in the air at levels too small to be seen in the measurements over the ground [3]. US Geological Survey (USGS) aerial measurements [4] show that the concentration of K, U, and Th vary a great deal throughout the United States, depending on the local rock or soil type. Manmade materials that contain natural materials such as asphalt, concrete, brick, tile, etc., also include $\mathrm{K}$, U, and Th varying in large amounts, depending on the origin of the source material $[5,6,7]$. Urban environments with raw materials brought in from different geologic areas can have a wide range of $K, U$, and Th values over small distances: streets may have been paved with materials from different quarries, and buildings consist of several materials from various locations.

Skyshine - the radiation emitted from the soil or other background sources scattered in the air before arriving at the detector - is the largest component of background measured at low energies $(<300 \mathrm{keV})$. 
Sandness [3] found when using a detector near the ground, the size of the computational model necessary to include all contributions from skyshine requires (1) a radius of $300 \mathrm{~m}$, (2) a soil depth of at least 1.25 $\mathrm{m}$, and (3) a height of at least $300 \mathrm{~m}$ of air above the soil.

For the MUSE project, benchmark-quality measurements of both sources and background were taken for the MUSE project during several campaigns at the Fort Indiantown Gap (FTIG) Combined Arms Collective Training Facility (CACTF) in Pennsylvania [8]. The facility includes twelve buildings, some with simulated damage, with several roadways arranged into a mock town. The CACTF is used by the military for urban training missions. For urban search measurements, this facility offers a small, wellcontrolled environment that remains the same over long periods of time. Scientists participating in the MUSE project were able to set up a variety of measurements without interrupting pedestrians or traffic since neither exist at the site. Detailed construction drawings of this site are available, so construction of a radiation transport model was easier than it would be for a street in a real city.

The measurements taken at FTIG were used to validate the models, methods, and data used in the virtual testbed. Once validated, the virtual testbed can be used to help plan experiments, interpret past measurements, evaluate detectors in different environments, and even create realistic data for detection algorithm development [9].

This paper focuses on comparing simulations to the FTIG measurements using CAD-based geometry models and traditional constructive solid geometry (CSG) models. Section II briefly describes the measurements taken at the FTIG CACTF. Section III details the modeling using both CAD-based and traditional CSG models. Section IV compares the simulations to the measurements, and Section V examines the ease and speed of using the two different types of model geometry.

\section{MEASUREMENTS}

To simplify recordkeeping and analysis, a coordinate system was applied to the FTIG site for the different measurement campaigns. As shown in Figure 1, the origin was set at the intersection of the centerlines of Road 3 and Road B, with the $x$-axis lying along the centerline of Road 3 and the $y$-axis lying along the centerline of Road B. Figure 2 shows the entire site and the detector positions for both the Road 3 and Road A measurements, as well as the Cs source positions used for the Road 3 measurements.

\subsection{ROAD 3 MEASUREMENTS}

In the September 2015 campaign at the FTIG CACTF, measurements of background radiation were taken with a $2 \times 4 \times 16$ in. $\mathrm{NaI}(\mathrm{Tl})$ detector at seven locations along Road 3. Measurements were also taken with an $81 \mu \mathrm{Ci}{ }^{137} \mathrm{Cs} /{ }^{137 m} \mathrm{Ba}$ source in one of four positions offset from the centerline of the road. At each source position, measurements were made at a subset of the seven detector locations. Some measurement positions had a clear line of sight between the source and the detector, and a few were obstructed. The detector and source positions and source are given in Table 1.

The detectors were $2 \times 4 \times 16$ in. $\mathrm{NaI}(\mathrm{Tl})$ detectors that were positioned on lightweight folding tables, with the detector center $84.8 \mathrm{~cm}$ above the road surface. Figure 3 shows the detector and associated equipment used by RSL-Andrews. Several similar setups were used to simultaneously measure background at different positions. Figure 4 shows measurements taken at positions $(30 \mathrm{~m}, 0 \mathrm{~m})$ and $(40$ $\mathrm{m}, 0 \mathrm{~m}$ ) along the centerline of the road. 


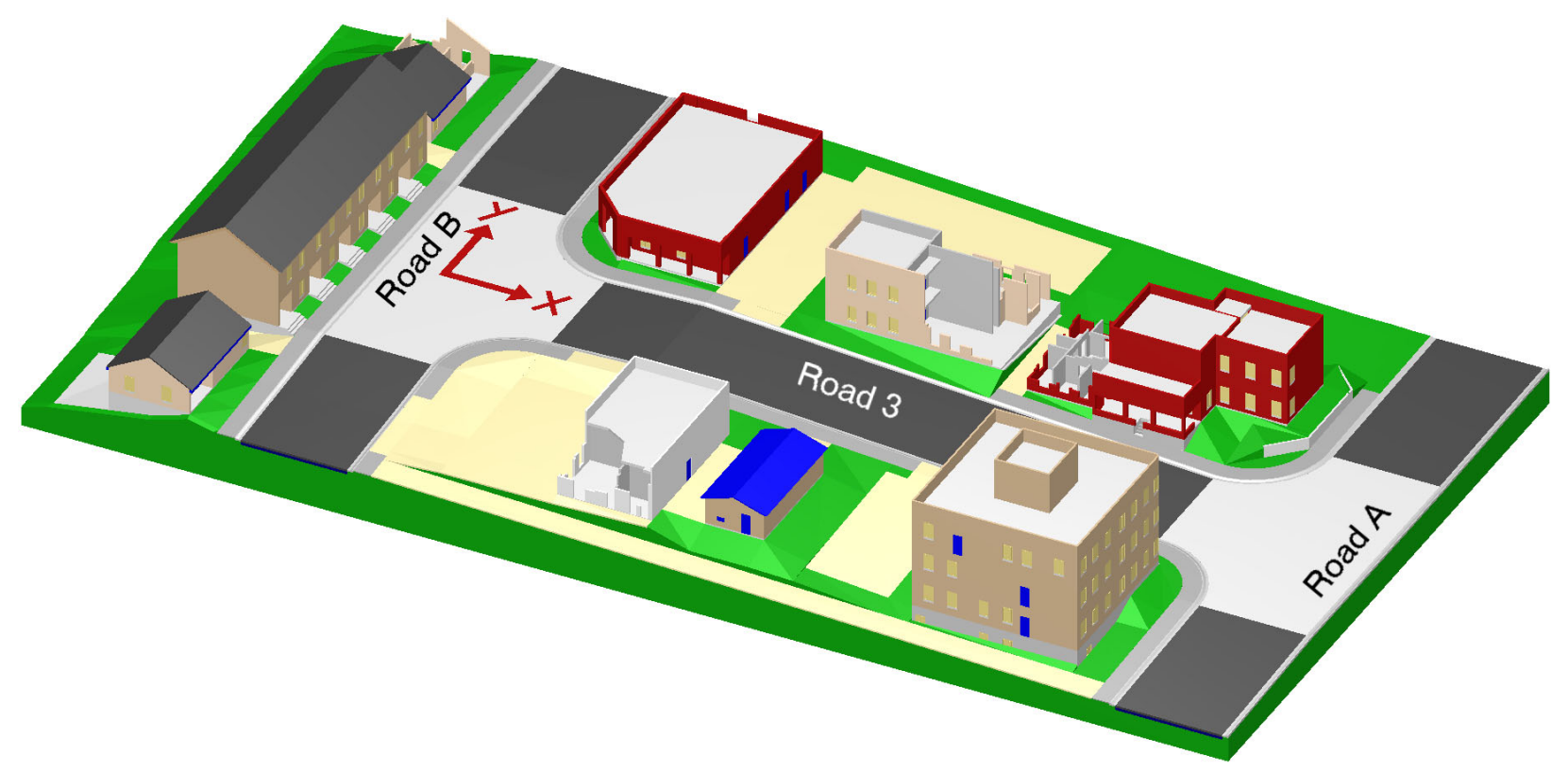

Figure 1. The coordinate system used for detector positions at the FTIG CACTF has its origin at the intersection of the centerlines of Road 3 and Road B. Buildings on the -x side of the origin are five connected townhouses with a single-family home on each side. One home has rubble. Along the $+y$ side of Road 3 are a onestory building with shops (red), a two-story office building with rubble (tan), and a 2-story police station with rubble (red). On the -y side of Road 3 are a one-story service station with rubble (white) with a large gravel parking area, a small storage building (blue roof) and a three-story hotel (tan).

\subsection{ROAD A MEASUREMENTS}

Measurements of background were taken along Road A of the FTIG site during the September 2016 campaign. The $2 \times 4 \times 16$ in. $\mathrm{NaI}(\mathrm{Tl})$ detectors in low-mass carts were used to hold all associated equipment for collecting radiation data, and the detector center was $53.1 \mathrm{~cm}$ above the road surface. Only background measurements were during this campaign. The detector and associated equipment are shown in Figure 5. Four carts were used to conduct the measurements across the FTIG site, as shown in Figure 6.

\subsection{MEASUREMENTS FOR BACKGROUND K, U, AND TH CONCENTRATIONS}

During the measurement campaigns of May 2015, August 2015, and September 2016, 70 high-purity germanium (HPGe) detector measurements were taken using an ORTEC Detective EX-100T. These measurements were taken of soil, asphalt, concrete (intersections, sidewalks, curbs, building foundations), gravel, and five colors of concrete masonry units (blocks) that had been used to construct the buildings. To reduce the interference from the surrounding materials, the HPGe detector was surrounded by a cave of lead bricks 2 in. $(5.24 \mathrm{~cm})$ thick. Swinney [10] used the measurements and detailed simulations of single-energy gammas distributed in layers to compute the concentrations $(\mathrm{Bq} / \mathrm{kg})$ of ${ }^{40} \mathrm{~K},{ }^{238} \mathrm{U} /{ }^{235} \mathrm{U}$ and their daughters, and ${ }^{232} \mathrm{Th}$ and its daughters in 11 different materials. The concentration of ${ }^{137} \mathrm{Cs}$ was also found for soil. 


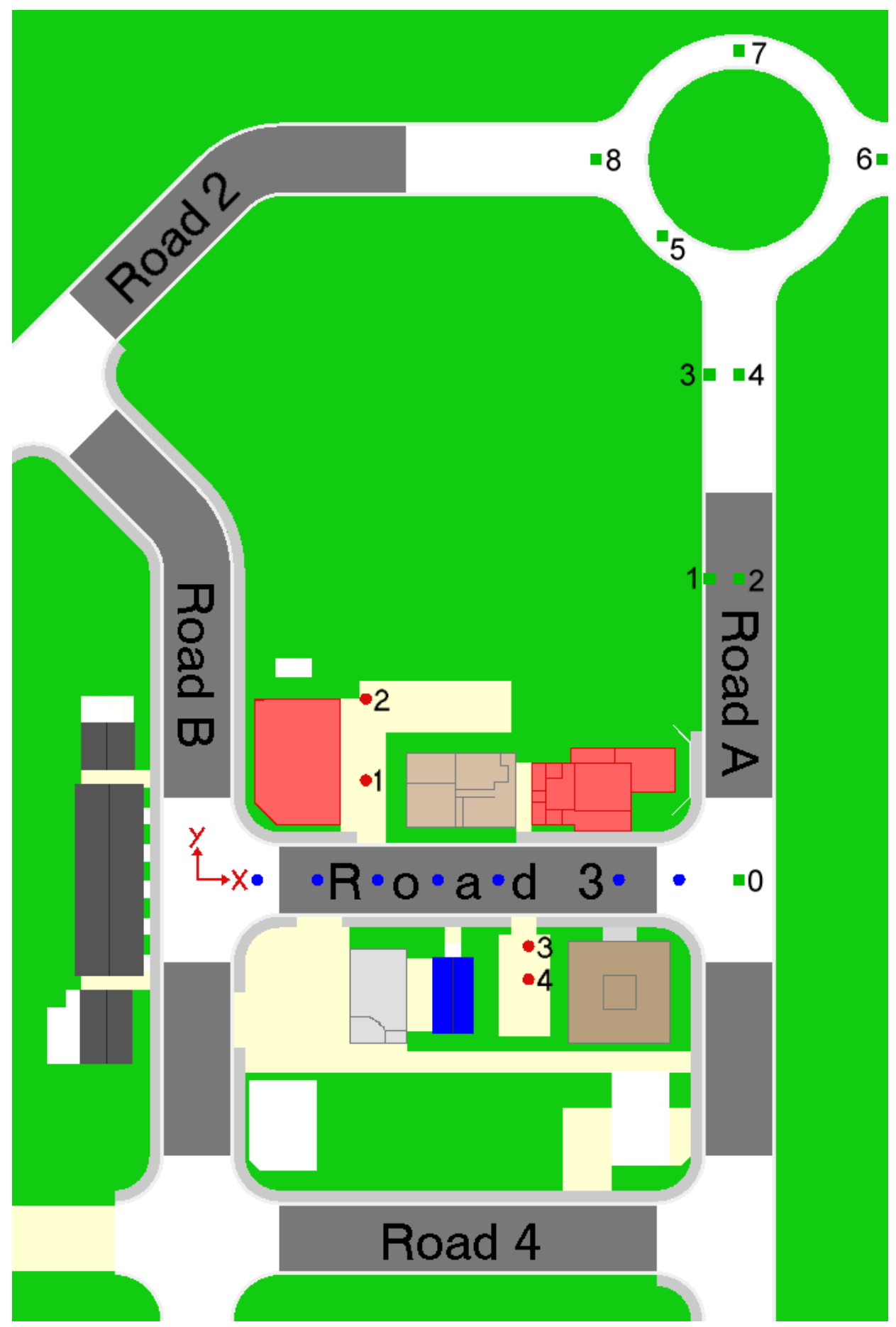

Figure 2. Overhead view of the main area of the FTIG CACTF with grass (green), asphalt roads (gray), sidewalks (light gray), gravel areas (light yellow), concrete (white), and buildings (red, blue, tan, dark gray, and brown). Road 3 detector locations are shown as blue circles and are labeled using their $\mathrm{x}$ coordinate value in meters (10 to 80$)$. Source positions used in the Road 3 measurements are shown as numbered red circles. Road A detector locations are shown as numbered dark green squares. 
Table 1. Detector and source locations for the Road 3 source measurements.

\begin{tabular}{|c|c|c|c|c|}
\hline Detector & \multicolumn{4}{|c|}{ Source Position (m) } \\
\hline $\begin{array}{c}\text { Coordinates } \\
\text { (m) }\end{array}$ & $\begin{array}{c}1 \\
(28,16.5)\end{array}$ & $\begin{array}{c}2 \\
(28,30)\end{array}$ & $\begin{array}{c}3 \\
(55,-11)\end{array}$ & $\begin{array}{c}4 \\
(55,-16.5)\end{array}$ \\
\hline$(10,0)$ & blocked & blocked & & \\
\hline$(20,0)$ & line-of sight & blocked & & \\
\hline$(30,0)$ & line-of sight & line-of sight & line-of sight & line-of sight \\
\hline$(40,0)$ & line-of sight & blocked & line-of sight & line-of sight \\
\hline$(50,0)$ & blocked & & & line-of sight \\
\hline$(70,0)$ & & & & line-of sight \\
\hline$(80,0)$ & & & line-of sight & blocked \\
\hline
\end{tabular}

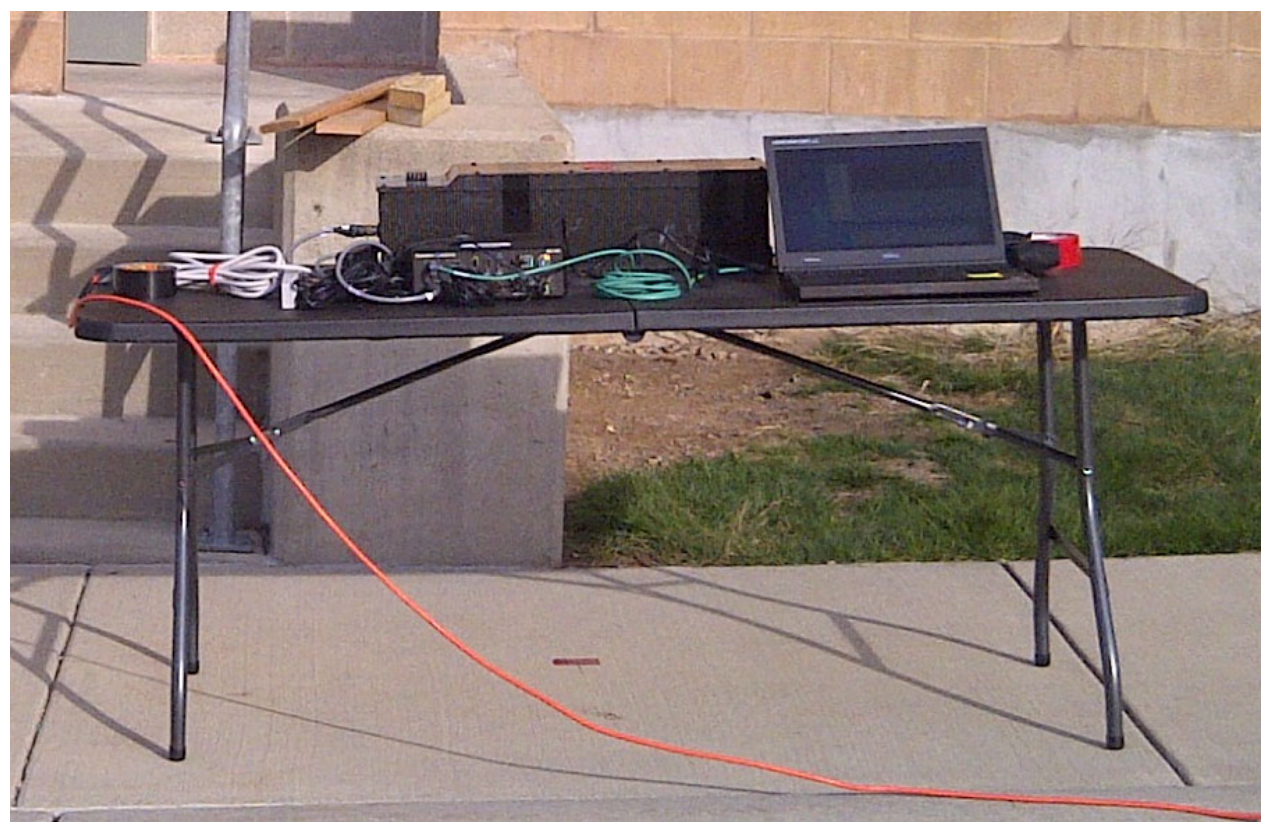

Figure 3. Experimental measurement setup based on an RSI detector used by RSL-Andrews. (Image courtesy of RSL-Andrews.) 


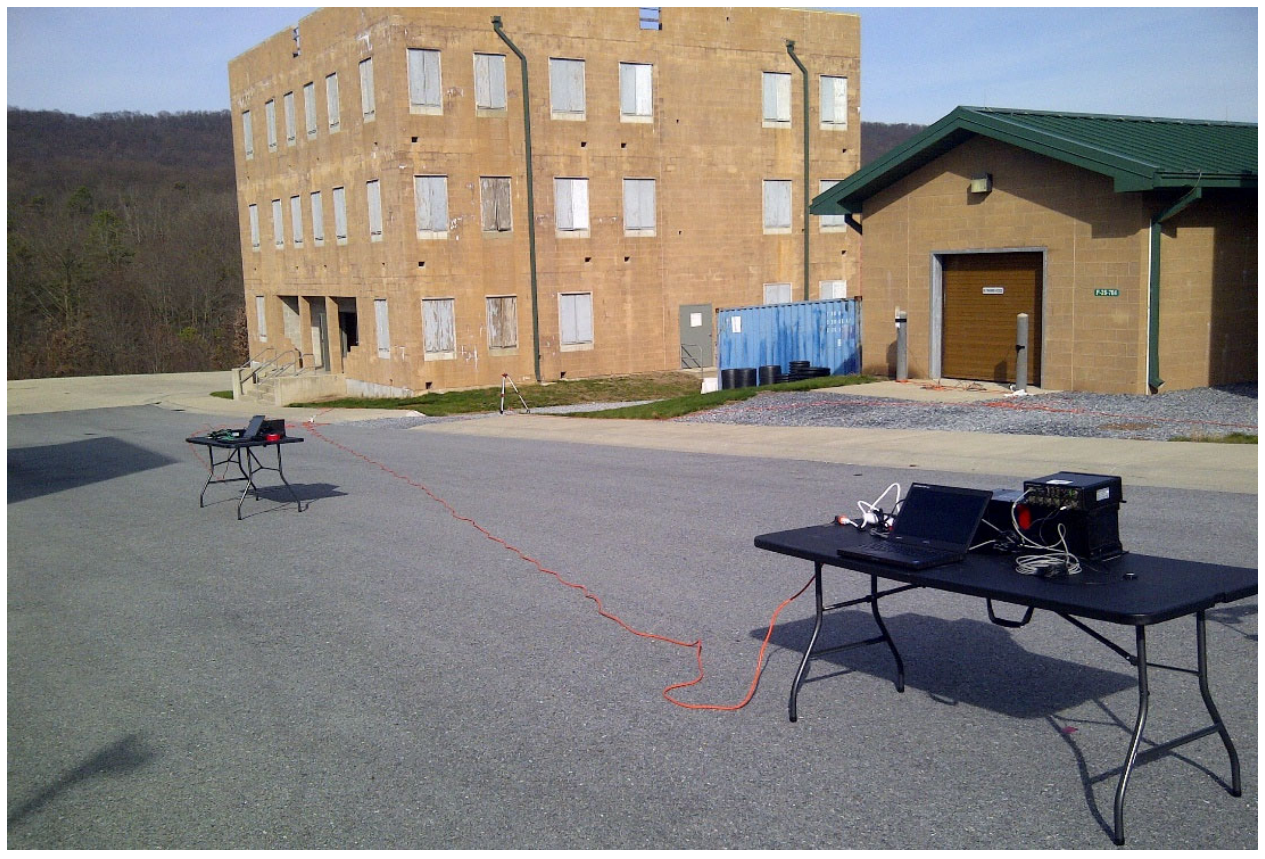

Figure 4. Measurements along Road 3 taken during the OPTUS-1 campaign used RSI $2 \times 4 \times 16 \mathrm{in}$. NaI detectors resting on a table. This image shows the measurement locations that are $40 \mathrm{~m}$ (left) and $30 \mathrm{~m}$ (right) from the intersection of the centerlines of Road 3 and Road B, which is to the right of the image. The tripod is at source location 3, with the Cs source 1 meter above the ground. The three-story hotel (left) and the small storage building (right) are also visible. (Image courtesy of RSL-Andrews.)

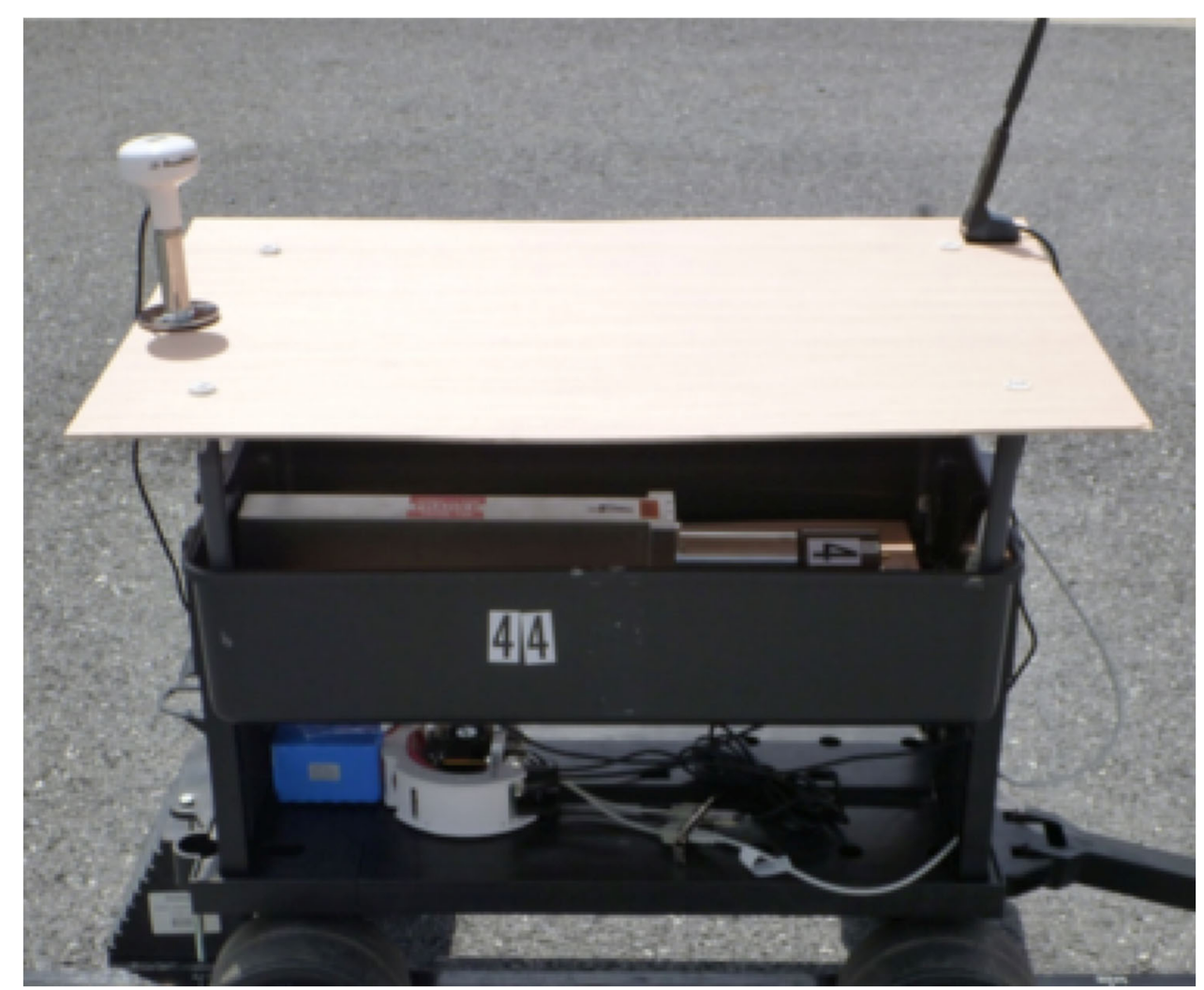

Figure 5. Detector in a cart with associated equipment, used by ORNL (Image courtesy of ORNL). 


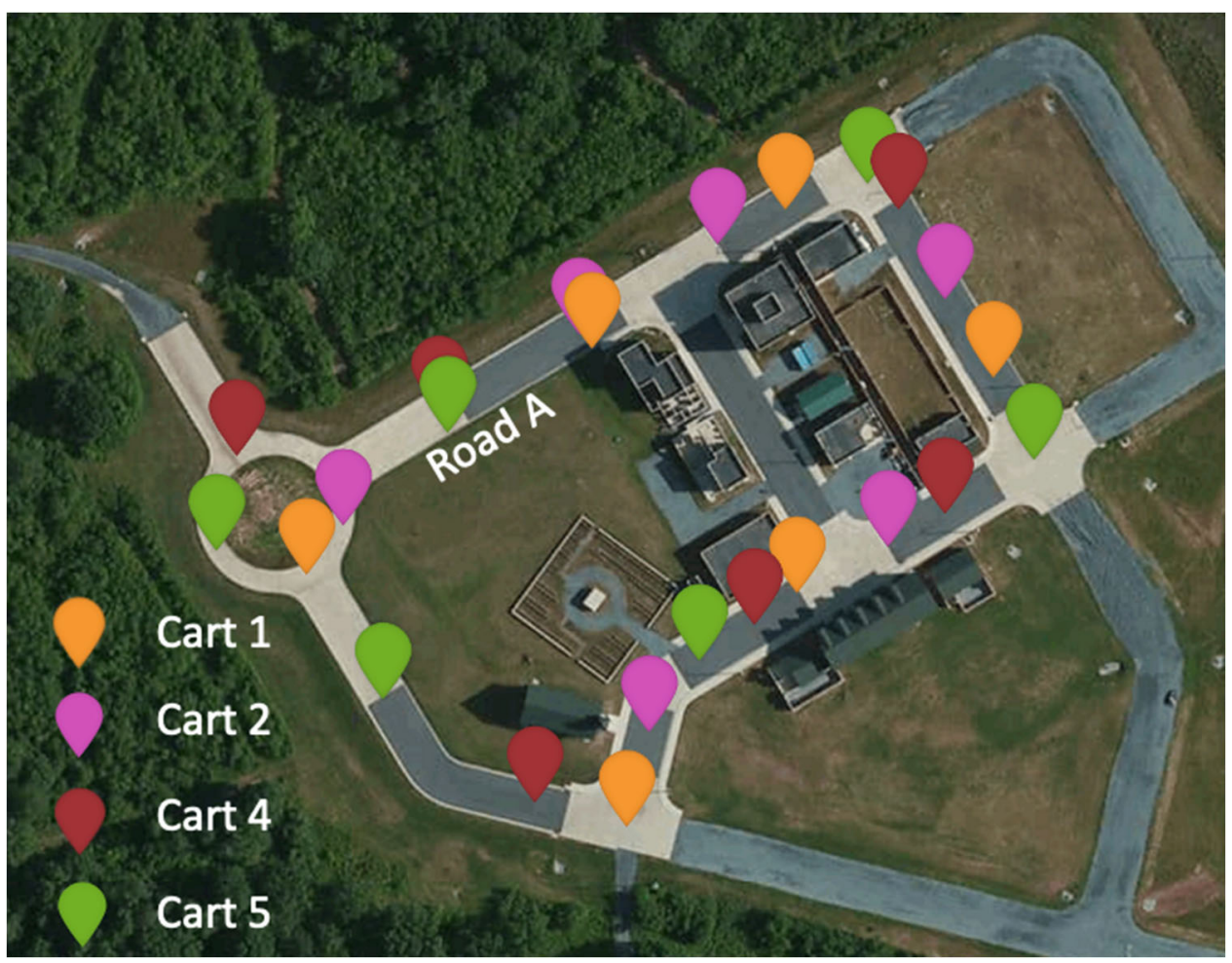

Figure 6. Locations of background measurements taken with the four ORNL carts. The Road A measurements from the police station to the circle are used in this paper (Image courtesy of ORNL).

\subsection{MEASUREMENTS USING A MOBILE RADIATION DETECTION SYSTEM}

During the September 2016 campaign, the project team fielded the Radiological Multi-sensor Analysis Platform (RadMAP) [11] to measure the FTIG facility. The RadMAP system synchronously collects multimodal data streams from three types of radiation detectors - two light detection and ranging (LiDAR) units, two Ladybug3 video camera units, a hyperspectral camera - as well as a vehicle odometry sensor, a weather station, and an inertial measurement unit (IMU). All of these were colocated on a truck. The measurement campaign lasted for multiple days, and numerous different data collections occurred, including static dwell, loops, and complete site surveys that were performed during different lighting conditions. One particular complete survey of the facility took 168 seconds. The LiDAR and IMU data from this survey are the subject of analyses based on RadMAP data described herein. Data from this $168 \mathrm{~s}$ survey are also the subject of study described in works by Bandstra et al. and Salathe et al.[12, 13]. The survey essentially comprised a figure-eight loop around the FTIG facility. The LiDARs are Velodyne high-definition LiDAR (HDL) 32E units mounted on the top-front corners of the RadMAP truck cargo box, with one LiDAR on the starboard side, and the other on the port side. The LiDAR units have a 41.33 degree vertical field of view ranging from -30.67 to 10.67 degrees from their horizontal axis, but they are tilted forward at $\sim 10.67$ degrees to achieve better coverage of the area in front of the vehicle. Further details on the RadMAP configuration are available in the article by Bandstra et al. [11]. 


\section{MODELING}

\subsection{THE SHIFT MONTE CARLO CODE}

Shift $[14,15]$ is a high-performance massively parallel Monte Carlo radiation transport code. It is part of a larger radiation code suite which also includes the Denovo [16] deterministic solver. Shift features both multigroup and continuous-energy physics in neutron, photon, and coupled neutron-photon modes, and it can be used to solve both fixed-source and $k$-eigenvalue problems. Shift features a multilevel parallelism scheme using the multiple-set overlapping-domain (MSOD) algorithm [17]. Space is decomposed into potentially overlapping rectangular cuboidal regions called blocks, and particle histories are decomposed across sets. In a problem with $N_{\mathrm{b}}$ blocks and $N_{\mathrm{s}}$ sets, the total number of parallel domains is given by

$$
N_{\mathrm{d}}=N_{\mathrm{s}} N_{b}
$$

Shift supports parallelism through message-passing interface MPI and by using threads with OpenMP, and it also features a graphical processing unit (GPU)-enabled solver [17].

\subsubsection{Hybrid Monte Carlo/Deterministic Capabilities in Shift}

Shift is coupled to Denovo to enable hybrid deterministic/Monte Carlo fixed-source simulations [18]. Denovo is a parallel deterministic transport code that features both $\mathrm{S}_{\mathrm{N}}$ and $\mathrm{SP}_{\mathrm{N}}$ solvers. Similar to Shift, it also decomposes the problem into blocks and sets. For the $\mathrm{S}_{\mathrm{N}}$ solver, the blocks are used in a KochBaker-Alcouffe (KBA) sweep algorithm [19]. Energy is discretized using the multigroup method, and the groups are decomposed across sets [20]. It is not necessary for the Denovo parallel decomposition to be the same as the Shift decomposition. Shift and Denovo can be run on different numbers of blocks and sets. However, it is currently a requirement that both Shift and Denovo be executed on the same number of parallel domains (which is the product of the number of blocks and sets) when running a hybrid simulation.

Shift features both the consistent adjoint-driven importance sampling (CADIS) [21] and the forwardweighted-CADIS (FW-CADIS) [22] hybrid methods, summarized here for completeness. The CADIS method is described first. Given a detector response function $\sigma_{d}(\vec{r}, E)$ spanning spatial region $V$ at position $\overrightarrow{\boldsymbol{r}}$ and energy $E$, the goal of a fixed-source Monte Carlo simulation is to calculate the detector response,

$$
R=\int_{0}^{\infty} \int_{V} \phi(\overrightarrow{\boldsymbol{r}}, E) \sigma_{d}(\overrightarrow{\boldsymbol{r}}, E) d \overrightarrow{\boldsymbol{r}} d E
$$

where $\phi(\vec{r}, E)$ is the flux. By setting the adjoint source to be equal to the detector response function,

$$
q^{\dagger}(\overrightarrow{\boldsymbol{r}}, E)=\sigma_{d}(\overrightarrow{\boldsymbol{r}}, E)
$$

and using the adjoint identity, the detector response $R$ can also be written as

$$
R=\int_{0}^{\infty} \int_{V} \phi^{\dagger}(\overrightarrow{\boldsymbol{r}}, E) q(\overrightarrow{\boldsymbol{r}}, E) d \overrightarrow{\boldsymbol{r}} d E
$$

where $\phi^{\dagger}(\overrightarrow{\boldsymbol{r}}, E)$ is the adjoint flux, calculated by solving the fixed-source radiation transport equation using the adjoint source given in Eq. (3), and $q(\vec{r}, E)$ is the forward source. Weight windows are then constructed such that 


$$
w_{\mathrm{c}}(\overrightarrow{\boldsymbol{r}}, E)=\frac{R}{\phi^{\dagger}(\overrightarrow{\boldsymbol{r}}, E)},
$$

where $w_{\mathrm{c}}$ is the weight window center. The source distribution is biased using

$$
\hat{q}(\vec{r}, E)=\frac{\phi^{\dagger}(\vec{r}, E) q(\vec{r}, E)}{R} .
$$

This method is ideal for optimizing for a single detector response. When multiple detector responses are desired, the FW-CADIS method is more appropriate. For this method, Eq. (3) is modified such that

$$
q^{\dagger}(\overrightarrow{\boldsymbol{r}}, E)=\frac{\sigma_{d}(\overrightarrow{\boldsymbol{r}}, E)}{\int \sigma_{d}(\overrightarrow{\boldsymbol{r}}, E) \phi(\overrightarrow{\boldsymbol{r}}, E) d E}
$$

to optimize for an energy-integrated response function, and

$$
q^{\dagger}(\overrightarrow{\boldsymbol{r}}, \mathrm{E})=\frac{1}{\phi(\overrightarrow{\boldsymbol{r}}, E)}
$$

to optimize for an energy-dependent response function. After this, Eqs. (4)-(6) are solved just as with the CADIS method.

\subsubsection{Computing the Background Radiation Source Using Shift}

When simulating background radiation, most materials in the problem become part of the source definition. The source definition also becomes very geometrically complex: as complex as the underlying geometry. To model the source, Shift overlays a Cartesian mesh on the problem geometry and uses its fast parallel ray tracer to compute the volume of each radioactive material in each grid cell. Combined with the density and specific activity of the material, the source strength of a given material within a given Cartesian mesh cell can be calculated. This can then be normalized to form a spatial distribution for the source. The source is sampled by first sampling a cell within the Cartesian mesh and then sampling a position within the cell. Positions in the cell are rejected if they lay outside the radioactive material. To simplify the calculation of the biased source, Shift requires that the Cartesian mesh used to discretize the source be the same as the mesh used for the Denovo calculation. This requirement could be relaxed in the future.

\subsubsection{Using CAD Geometry in Shift}

Shift communicates to geometry packages through a generic interface. Any geometry package which satisfies that interface can be used with Shift, and Shift currently features a variety of supported geometries, including SCALE geometry [23], MCNP geometry [24], the Reactor ToolKit geometry (used to model pressurized water reactors for the Virtual Environment for Reactor Applications [VERA] software package [25]), and CAD geometries through an interface to the Direct Accelerated Geometry Monte Carlo (DAGMC) library [26]. DAGMC works by tessellating the geometry using a triangular surface mesh and tracking through that mesh.

Most of the primitive geometry functionalities - such as computing the distance from a position to a geometric surface or finding the cells that bound a surface - are provided directly by the DAGMC library. The Shift interface supplements DAGMC with an additional capability for fast initialization of particles. When particles are sampled from the source distribution, only the particle's position within the geometry is known. During initialization, Shift interrogates the geometry to compute the bounding boxes for all 
geometric cells and loads these bounding boxes into a $k$-d tree. This enables Shift to rapidly find the cell containing a given position at the start of a particle's history.

\subsection{THE CSG MODEL}

The CSG model of the FTIG site was developed for the KENO and MAVRIC sequences in the SCALE package [23] and was based on the construction drawings supplied by the US National Guard. The SCALE input files are human-readable text files and can be used by Shift to define its geometry, as well. The CSG model shown in Figure 7 and used in this study is similar to one used in a previous study [27] but has been extended beyond the Road 3 area to include Road 2 and an extension of Road A to the circle roundabout, where the Road A measurements were taken. The origin and axes used in the CSG model correspond to the coordinate system used during the measurements. Note that Figure 1 and Figure 2 shown earlier were made from the CSG model. This model required several person-months to complete.

The roadways are made of asphalt or concrete segments that are $36 \mathrm{ft}(10.97 \mathrm{~m})$ wide, and these are inside a set of curbs that are $2 \mathrm{ft}(0.61 \mathrm{~m})$ wide, except at the roundabout, where the road is only $15 \mathrm{ft}(4.57 \mathrm{~m})$ wide. Some roadways are lined with sidewalks that are $6 \mathrm{ft}(1.83 \mathrm{~m})$ wide. The intersections are made of concrete and extend $45 \mathrm{ft}(13.72 \mathrm{~m})$ outward from their centers (the intersection of the centerlines of the two roadways). The concrete roadways are made of a layer of concrete 10 in $(25.40 \mathrm{~cm})$ deep on top of a 4.5 in $(11.43 \mathrm{~cm})$ base layer of coarse aggregate. The asphalt roadways have a 4.5 in $(11.43 \mathrm{~cm})$ layer of asphalt, a 4 in $(10.16 \mathrm{~cm})$ base layer of aggregate, and an 11.5 in $(29.21 \mathrm{~cm})$ subbase aggregate layer.

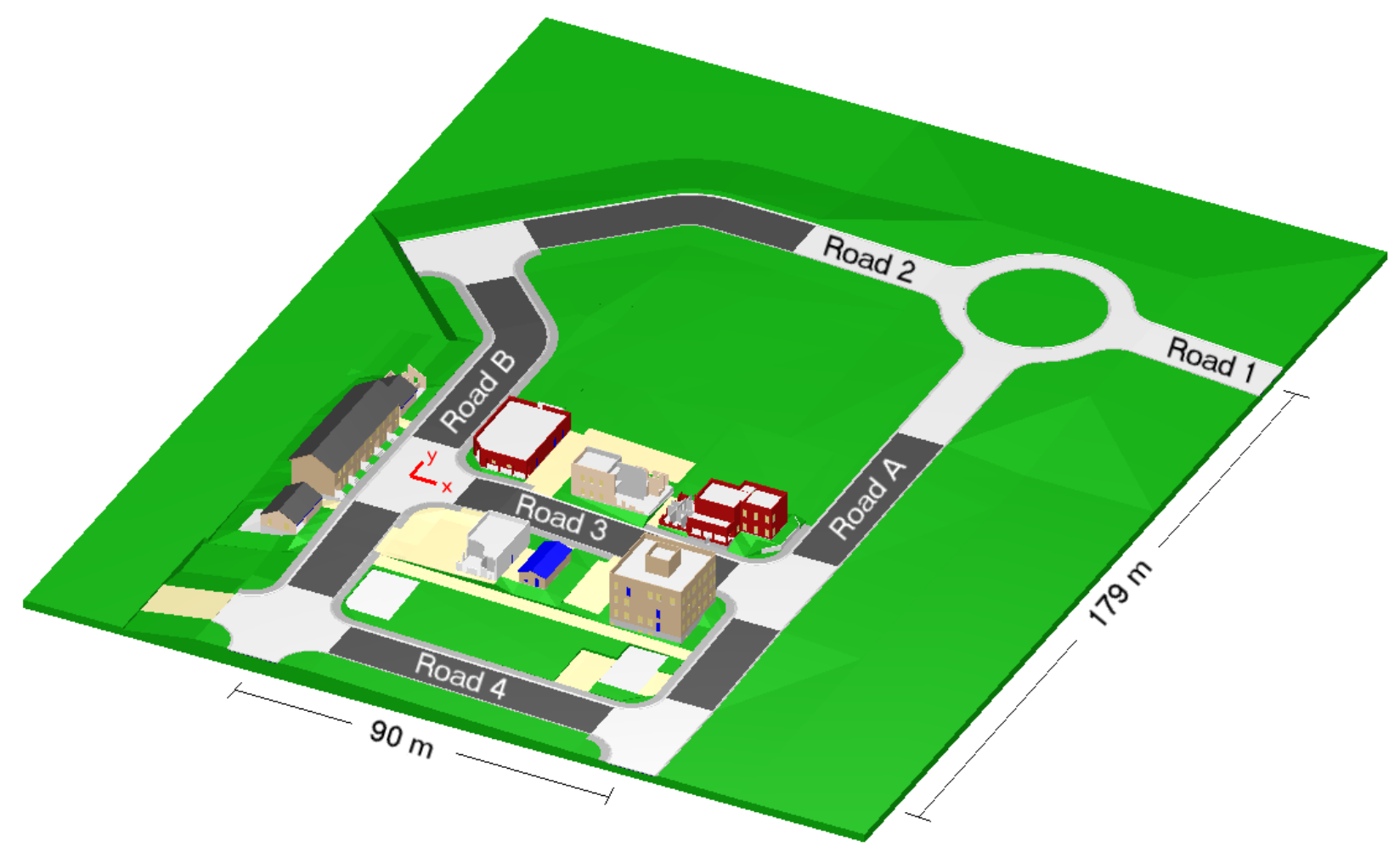

Figure 7. The CSG model of the FTIG viewed from the northeast. Included are asphalt roads (gray), concrete intersections and roads (white), gravel areas (light yellow) and nine buildings (light gray, red, blue, tans). Two buildings along Road 4 and a church at the intersection of Road B and Road 2 were not included in the model. The distance between the centerlines of Road A and Road B is $295 \mathrm{ft}(89.92 \mathrm{~m})$, and the distance from the centerline of Road 4 to the center of the roundabout is $587 \mathrm{ft}(178.92 \mathrm{~m})$. Extra soil was included $500 \mathrm{~m}$ outward in each direction from the main area, as well as $500 \mathrm{~m}$ of air above. Soil depth varied with the terrain but was at least $2 \mathrm{~m}$ thick. 
Curbs drop down 4 in $(10.16 \mathrm{~cm})$ from the sidewalk level to the road surface. The gravel roadways and parking areas near the buildings are 6 in $(15.24 \mathrm{~cm})$ thick. To make connections between the roadways smoother, the road surfaces only slope in either the $x$ - or $y$-direction (not both), and the intersections and roundabout are level. Elevations for the roadways and the terrain shown in Figure 1 and Figure 7 were based on the measured elevations in the construction drawings.

Nine buildings are included in the CSG model. All buildings at FTIG used 8 in $(20.32 \mathrm{~cm})$ concrete blocks. The garage/service station with rubble, a small storage building, and a three-story hotel are along the $-y$ side of Road 3. The $+y$ side of Road 3 includes a building with three small retail shops, a twostory office building with rubble, and a police station with rubble. The retail shops include pillars made of 12 in $(30.48 \mathrm{~cm})$ blocks. Five townhomes are along Road B, as well as single-family homes on each side, one with rubble. The buildings include the wooden doors and windows, but only the outer shells are modeled, except for the hotel, for the interior floors and walls are modeled. A fast-food restaurant, a large courtyard, and a firehouse along Road 4 and a church near the intersection of Road B and Road 2 are not included in the model because they are far from the measurement locations. To accurately model the skyshine, $500 \mathrm{~m}$ of additional soil was added outward in each direction from the main area, as well as 500 $\mathrm{m}$ of air above. Soil is modeled to be at least two meters deep, and it varies in depth over the site due to changing terrain elevation.

\subsection{CAD MODEL}

A labeled three-dimensional (3D) point cloud was produced using RadMAP's contextual sensor data. Accelerometer and gyroscope data from RadMAP's Inertial Navigation System (INS) and point cloud data from the two LiDAR units mounted on the front of the vehicle were input into Google Cartographer [28], an opensource software for performing simultaneous localization and mapping (SLAM). The data collection chosen was a 165 -second collection in which the vehicle drives continuously on all roads around the FTIG facility. This contiguous dataset was selected to represent a realistic vehicle-based measurement of an urban environment where minimum and maximum vehicle speeds are constrained and practicable 'coverage' of the streets, whereby the measurement consisted of one or two passes of each road segment. The output of SLAM is a pose graph solution of the vehicle as a function of time in a fixed frame and a collection of all LiDAR points mapped into the fixed frame.

For the same data set, the panoramic images from the two Ladybug3 cameras were semantically labeled with 11 classes using random forest classification of image superpixels [12]. The classes used were grass, forest, building rooftop, concrete, asphalt, gravel, sky, vehicles, and red, brown, and gray buildings. The semantic labels of these images were then projected into the point cloud using the SLAM solution and relative positions and orientations of the cameras. Backprojection of each pixel of each image was performed by examining the 3D spherical wedge subtended by the pixel and stepping outward in increments of $1 \mathrm{~m}$ until points were identified within each $1 \mathrm{~m}$ thick wedge. The points within that wedge were then labeled according to the material classification of that pixel, and no further labeling was performed using that pixel. Any pixel could be labeled by multiple images in the data set, so a histogram of labels was kept for each pixel, and the most frequently used label was assigned at the end. This labeling procedure is described by Salathe et al. [13], and the results are shown in Figure 8. 


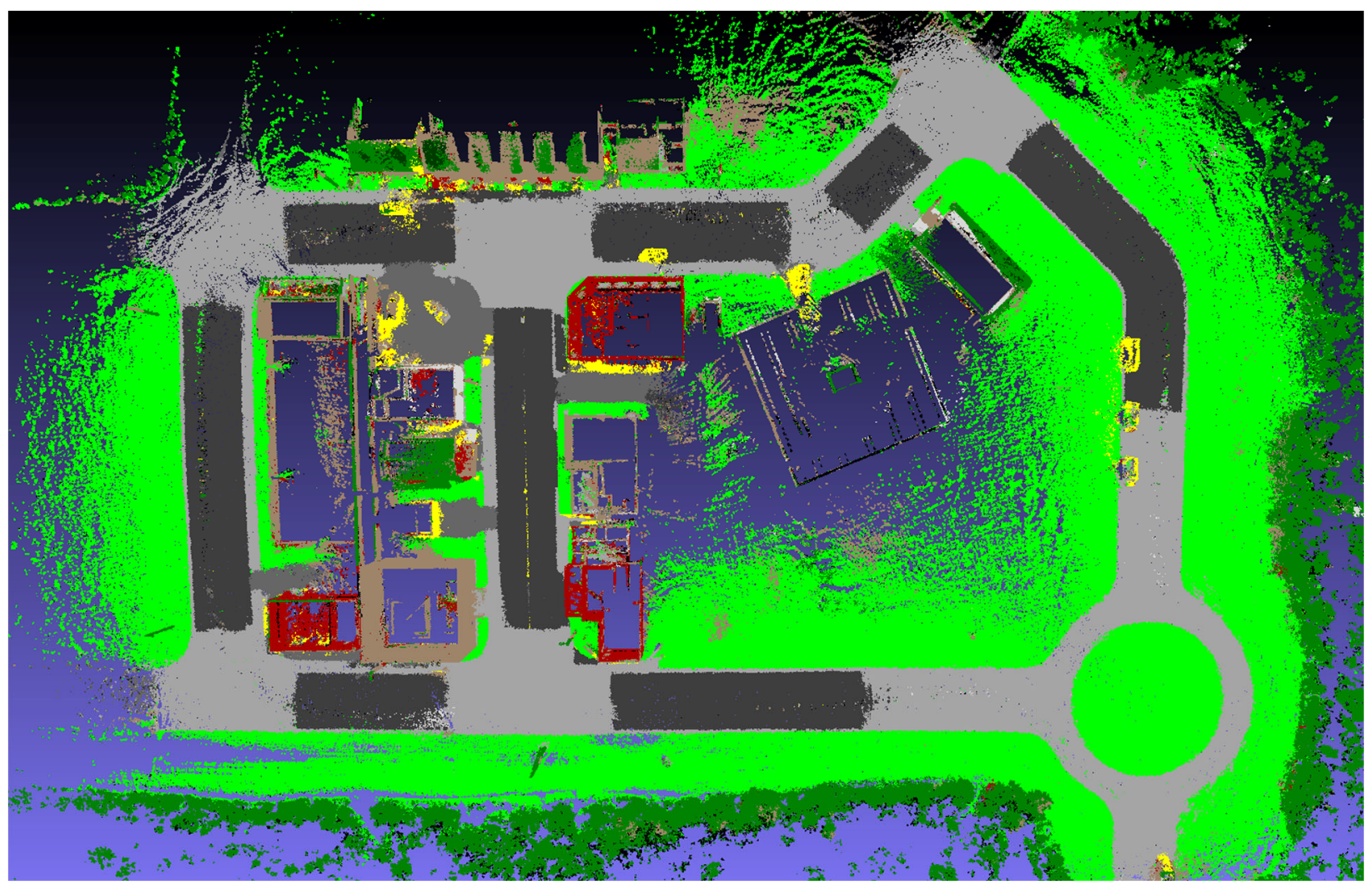

Figure 8. Top-down view of the semantically labeled point cloud produced by RadMAP during $165 \mathrm{~s}$ of driving around the FTIG facility. The point cloud was produced using SLAM, and the labels are from the semantically labeled images from RadMAP's panoramic cameras, which were produced using a machine learning classification tool trained on custom-labeled data from FTIG.

A semiautonomous workflow was created to convert the semantically labeled point cloud of the buildings and terrain into a collection of CAD models. Each CAD model is a dimensionally accurate solid model of a unique object in the point cloud, and the global reference is preserved. The composite of all CAD models provides a complete model description of the environment. The point cloud was first downsampled to a grid size of $0.3 \mathrm{~m}$, and isolated points were removed through denoising. The point cloud was then split into two collections of points corresponding to the terrestrial surface and objects on the surface. A two-dimensional (2D) local linear regression was used to generate a surface fit to the terrain points on a predefined 2D grid in the horizontal plane. This surface was then extruded downwards multiple meters in the open-source FreeCAD software to forge a solid model representation of the terrain. An orthographic overhead projection of the segmented terrain points was then converted to an image, and the boundaries of the various segmented regions (representing distinct materials) were estimated with closed polygons. As is often required, the boundaries of the segmented image were manually merged and simplified. These boundaries were then extended downward, and the closed surfaces were used to iteratively carve the complete terrain block into separate CAD models with distinct material assignments.

CAD models for objects on top of the surface were then derived from the companion non-terrain point cloud. This algorithm only includes buildings, although trees and other clutter could be added. The point cloud was first filtered by calculating local normal vectors and preserving only point clusters with normal vectors in the horizontal plane. The filtered cloud was then subjected to a clustering algorithm to separate collections of points corresponding to individual buildings. Each point cluster was then profiled in the horizontal dimensions through subsequent cuts of the vertical range subtended by the points. Each horizontal profile of a building used boundary regularization to achieve a concise, dimensionally accurate, 
closed polygon fit for that layer. After all layers were profiled, similarities between neighboring horizontal profiles were used to detect flat vertical components. A summary description of the profiles over defined vertical ranges was then extruded into a solid CAD model representation of the building or object. Finally, the individual objects were then extended downwards by a few meters, and a Boolean operation between the object and the terrain CAD models was used to "excavate" the terrain and ensure flush positioning of the objects and the terrain.

A method for converting CAD models extracted from LiDAR into Shift-readable CAD files was created. This method involves processing extracted CAD models which usually start as *.step or *.sat files for the separate objects populating an environment. These common file types can be modified and converted into other file types using any standard CAD software, such as SOLIDWORKS, Autodesk or Creo. The CAD model generated from LiDAR measurements of FTIG was processed using SOLIDWORKS. When imported into a commercial CAD software, the generic *.step or *.sat file from the extraction process was converted into the software's proprietary file format so that the model could be modified.

Within the CAD software, steps were taken to improve the accuracy of models generated from LiDAR, such as combining multiple extracted CAD models into a single model, manually adding small features which were not captured within the semi-automated extraction process, and extending air and soil volumes to better account for groundshine and skyshine. Figure 9 shows the additional large volumes added to the extracted FTIG CAD model within SOLIDWORKS. After the model was modified within the commercial CAD software, the file was converted back to a *.sat file and was exported out of the CAD software for transport preprocessing.

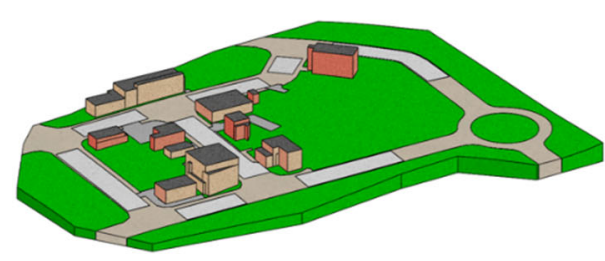

LiDAR

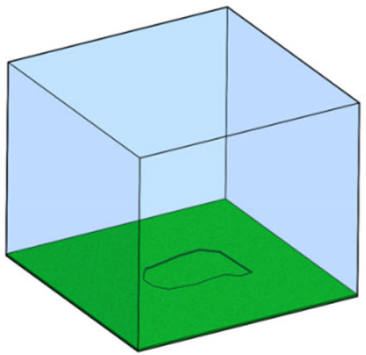

Extra Air, Soil \& Graveyard

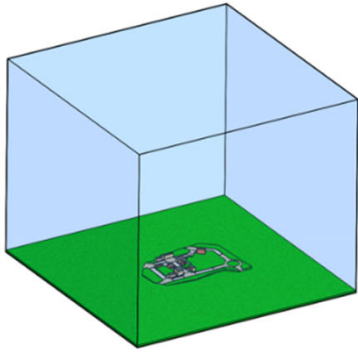

Finished CAD

Figure 9. SOLIDWORKS process visualization for the FTIG model. A large volume of air and large amounts of soil were added to the main LiDAR representation of the FTIG site to properly account for skyshine.

A preprocessing utility in the Python [29] scripting language was developed to convert data generated by the LiDAR postprocessing to the format that Shift can read. The utility script communicated with CUBIT [30] via its Python interfaces to reconstruct model geometry, and it utilized the DAGMC [31, 32] plugin to export the final model using the mesh-oriented database $[33,34]$ (in H5M file format). This database is used by Shift for particle tracking during transport calculations. The utility was developed to automate the workflow as much as possible by using a common interfacing method-Python interfaces in this caseso that overall modeling efficiency can be improved and errors can be minimized.

The data to the script were provided in different formats for each layer: the terrain in facets format, the roads and similar pavement features in a vertex-populated surface points format, and buildings in a profile-based format with corner vertices and elevation of each profile. All layers were processed via the script using CUBIT Python functions to carve out the current layer from the previous ones, ensuring watertight seals between each layer connection. First, the terrain was reconstructed by creating a surface using the facets which were extruded to a user-defined depth (set as $10 \mathrm{~m}$ ) to represent the soil as the 
main layer in the geometry. Next, the roads and pavements were all constructed individually by creating shapes using shape definitions (e.g., prism, cylinder, or sphere) and parameters supplied either by the data or the user, such as material and thickness. For example, concrete slabs have a depth of $25.4 \mathrm{~cm}$ and asphalt has a depth of $11.43 \mathrm{~cm}$. Each modeled layer of road or pavement was then carved out from the terrain. The building models were then reconstructed one by one, with multiple profiles, each profile representing a floor. User parameters for buildings included wall thickness (set as $20 \mathrm{~cm}$ ), with the remaining volume filled with air, along with material assignments for the walls. After reconstruction, each building was also carved out from previous layers of terrain, roads, and pavements. Finally, ample amounts of air (with a height of $500 \mathrm{~m}$ ) and soil (spanning $500 \mathrm{~m}$ in each direction) were added to the model to account for groundshine and skyshine phenomena by introducing a slab of extended soil and a prism layer of air above the terrain. The real model entities were carved out from the added air and soil layers to ensure a watertight seal on layer boundaries. Illustrations of each model layer and reconstruction are shown in Figure 10 and Figure 11, respectively. The script checked and validated the model before it was exported to an H5M file. Before running the actual particle transport calculations, which can be computationally expensive to investigate model issues, the H5M was also visually inspected by quickly rendering it with the ray tracer used by Shift.

Although it took a long time to develop the above workflow, if a new CAD model for Shift of a similar site were to be created, it is estimated that it would require 12 hours of processing after the initial measurement.

\subsection{OTHER MODELING TOPICS}

Material compositions for both the CSG and CAD models were taken from standard composition tables in SCALE and from the PNNL materials compendium [35]. Material compositions for carbon steel doors and some roofs and for dry air were taken from SCALE. Concrete roads, intersections, curbs, sidewalks, blocks for the buildings, building foundations, and stairs all used PNNL's "Concrete, Ordinary (NIST)" material composition. Concrete block walls were reduced in density to account for the air cavities. Gravel for the parking areas used PNNL's "Rock (Average of 5 Types)" at 75\% of the listed density (a reasonable guess) to account for loose packing. When used as the base layers under the road, the listed density was only reduced by $5 \%$ (to account for compaction during construction). The soil was modeled using "Earth, U.S. Average" and asphalt was modeled using "Asphalt Pavement." Plywood in the townhouse and the single-family home roofs, as well as the wooden windows installed in most buildings, were modeled using "Wood (Southern Pine)."

The concentrations for the NORM materials used in computing the background/skyshine component were taken from a MUSE paper by Swinney et al. [10]. These values are the averages of different measurements for the given material at the FTIG site. Sixteen materials with NORM concentrations were present in the CSG model, and seven materials were present in the CAD model. Fewer materials were involved in the CAD model due to the classes specified in the random forest classification that started the process of creating the model. 


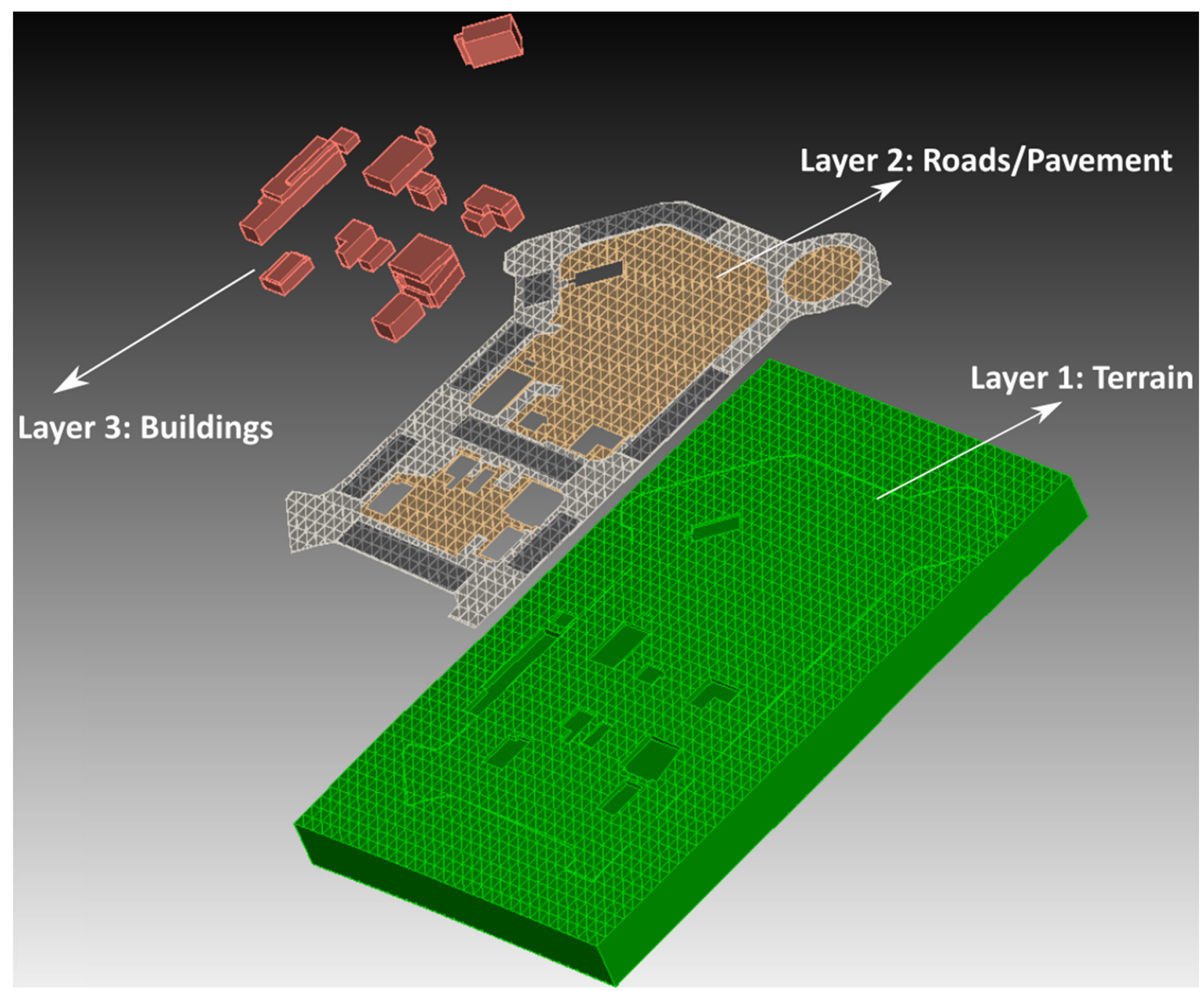

Figure 10. Three layers used to build the CAD geometry.

The detector response functions (DRFs) depend on factors including, but not limited to, detector type, material, geometry, operating conditions, and emitted radiation characteristics. The $\mathrm{NaI}(\mathrm{Tl})$ detector used in the FTIG measurements, measuring $2 \times 4 \times 16$ inches, does not have an isotropic DRF, mainly because of the significant variations in the surface area and penetration depth, depending on the direction of incoming particles. A directional detector response function (DDRF) [36] was used to calculate the count rates of the detector with the fluxes and directional currents computed by Shift. The DDRF was generated using a set of discretized quadratures established around the detector in which each quadrature stores a set of DRFs spanning the possible energy spectrum. The DDRF database for the detector was created in advance and is independent from the simulation models, providing a portable, reusable database for any other application in which the same detector is used in the simulations (this model of NaI detector is many applications). Moreover, since the detector itself is not modeled in the simulations, the DDRF also provides the flexibility to place the detector at any desired location in the real model. Region and mesh tally definitions in Shift have been updated to store partial currents for the cardinal directions $\left(J_{-\hat{x}}, J_{+}\right.$, $\left.J_{-\hat{y}}, J_{+\hat{y}}, J_{-\hat{z}}, J_{+\hat{z}}\right)$. These definitions were used later in a postprocessing tool to interpolate DDRFs to calculate the count rate on a given region or mesh. 


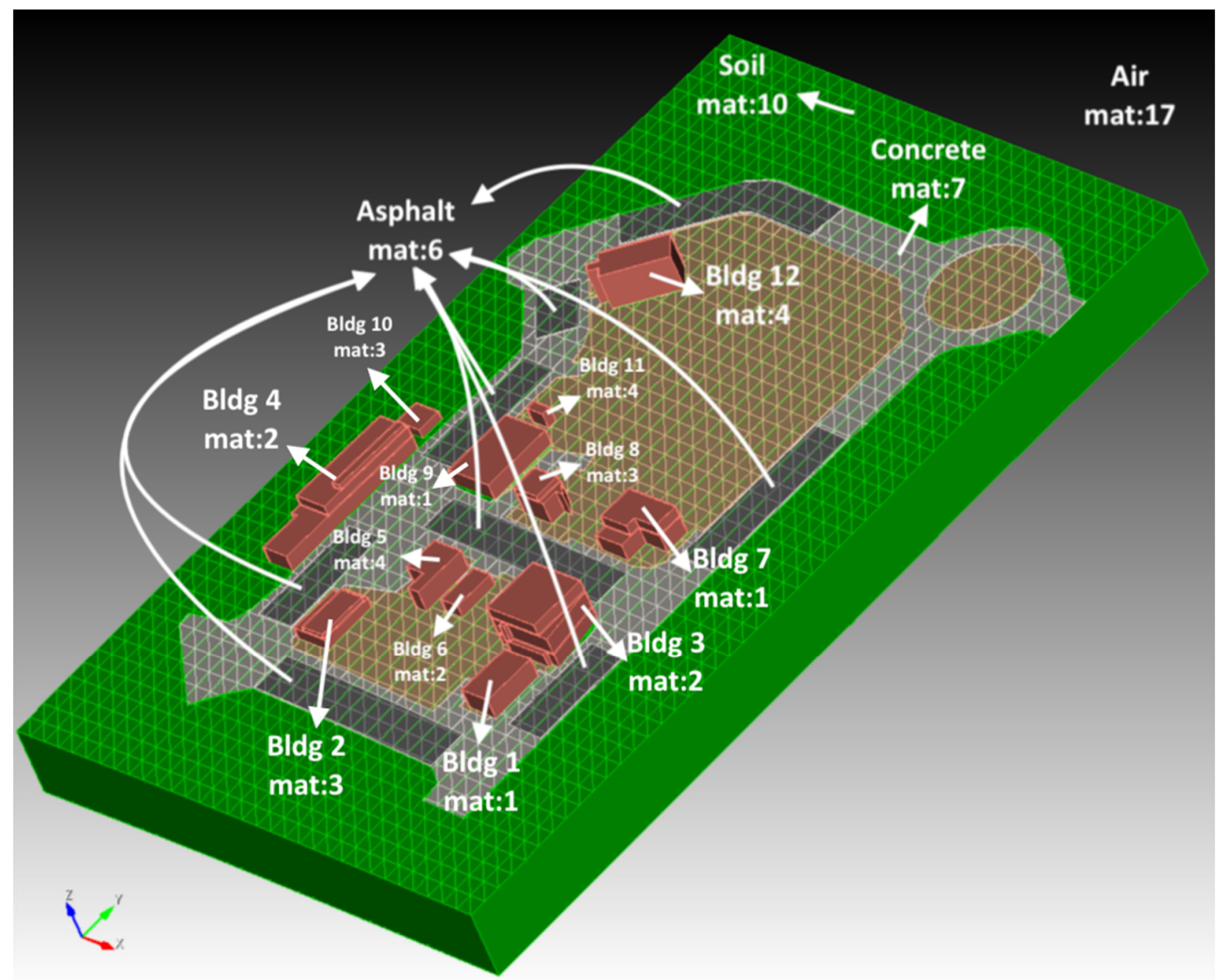

Figure 11. The FTIG CAD model with all layers merged and materials assigned. About $500 \mathrm{~m}$ of air above and soil outward in each direction were included in the model but are not shown in the figure. 


\section{COMPARISON OF SIMULATIONS TO MEASUREMENTS}

Twelve simulations were performed, six using CSG, and six using the CAD geometry. These cases were the background component optimized for the Road 3 measurement locations: one case for each Cs point source for the Road 3 measurements, and the background optimized for the Road A measurement locations. The FW-CADIS method [22] was used to optimize the calculation of flux at the detector locations. This method began by raytracing the geometry to create a mesh geometry for the Denovo discrete ordinates solver [16]. The mesh used voxels that were $100 \times 100 \times 30 \mathrm{~cm}$ in the main portion of geometry (streets and buildings) and larger voxels further away from the center of the model. The mesh was $216 \times 255 \times 76$. For the background calculations, the same mesh was used to construct the mesh source. A forward and an adjoint calculation were performed with Denovo to create an importance map and source biasing parameters to optimize the flux tallies at each detector location. The Shift Monte Carlo code used the original geometry (CSG or CAD), the biased version of the source (biased in energy for the point sources, biasing in coupled space/energy for the background), and the space/energy importance map. The flux tallies were single-voxel mesh tallies of $100 \times 100 \times 50 \mathrm{~cm}$ around the detector locations. After Shift completed the calculation, the count rate spectra for each detector were created using the python utility that processed the energy-dependent fluxes and partial currents into pulse-height spectra, and an energy resolution function $(7.5 \%$ at $662 \mathrm{keV}$ in this work, chosen to match previous measurements with the detectors) was also applied.

Each simulation ran for about 25 hours on 32 processors on an AMD Opteron 6378 central processing unit (CPU). The Denovo times were much shorter than the Shift Monte Carlo times: 9 and 13 minutes for the forward and adjoint calculations for the CSG with point sources, 16 and 13 minutes for the forward and adjoint calculations for the CSG background, 12 and 15 minutes for the forward and adjoint calculations for the CAD with point sources, and 18 and 15 minutes for the forward and adjoint calculations for the CAD background. In the Monte Carlo portion of the calculations, each simulation ran for about 24 hours. The long run times were chosen to reduce statistical uncertainties enough that a spuriously high flux bin did not appear as a physical peak in the final processed count rate spectrum.

\subsection{ROAD 3 BACKGROUND}

Background was measured at seven locations along the centerline of Road 3 at 10, 20, 30, 40, 50, 70, and $80 \mathrm{~m}$ from the origin of coordinates. Locations at 10 and $80 \mathrm{~m}$ were over concrete intersections, and the locations in between were over asphalt. Examples for two background measurements are shown in Figures 12-15 for locations at 10 and $40 \mathrm{~m}$ from the origin. For each location, all spectra are shown for the measurement, the CSG simulation, and the CAD simulation. Uncertainties are not shown in these figures because the high uncertainties with the measured data $(<5 \%$ for $0-500 \mathrm{keV}, 5-10 \%$ for $500-1000$ $\mathrm{keV}, 5-15 \%$ for $1000-1500 \mathrm{keV}, 10-50 \%$ for $1500-2000 \mathrm{keV}$, and $15-100 \%$ for above $2000 \mathrm{keV}$ ) would obscure the other curves. Plots are provided for each location showing close-up views of the ${ }^{40} \mathrm{~K}$ peak and low-energy region, which is dominated by skyshine. Statistical uncertainties of the Monte Carlo simulations were typically $1-4 \%$ below $2000 \mathrm{keV}$ and up to $8 \%$ above $2000 \mathrm{keV}$.

For the most part, the CSG and CAD simulations overlaid each other. Note that the simulated results were not normalized; the magnitudes of the count rate spectra are only a function of the concentration values of the $\mathrm{K}, \mathrm{U}$, and Th used in the various materials in the models and the transport. The model results are from first principles. Results for the other locations were similar. In a couple of instances, the CSG and CAD simulations disagreed by a few percent in either the ${ }^{40} \mathrm{~K}$ peak or in the low-energy/skyshine region, but these cases had high levels of statistical uncertainty for one or the other simulation in those channels. 


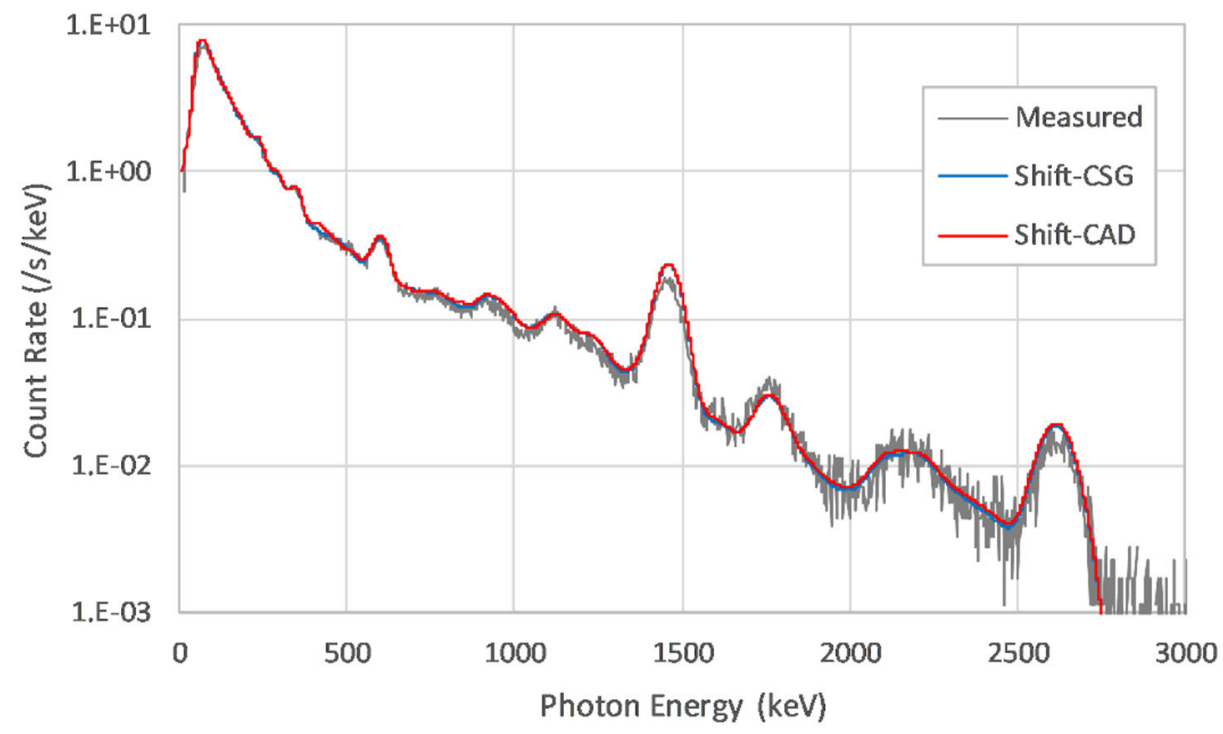

Figure 12. Road 3 background count rate spectra at location $x=10 \mathrm{~m}$ with measurement data and both CSG and CAD simulations. The CSG and CAD simulations agree very well, and both have good agreement with the measured data. Some differences are apparent at the ${ }^{40} \mathrm{~K}$ peak and in the low-energy region.
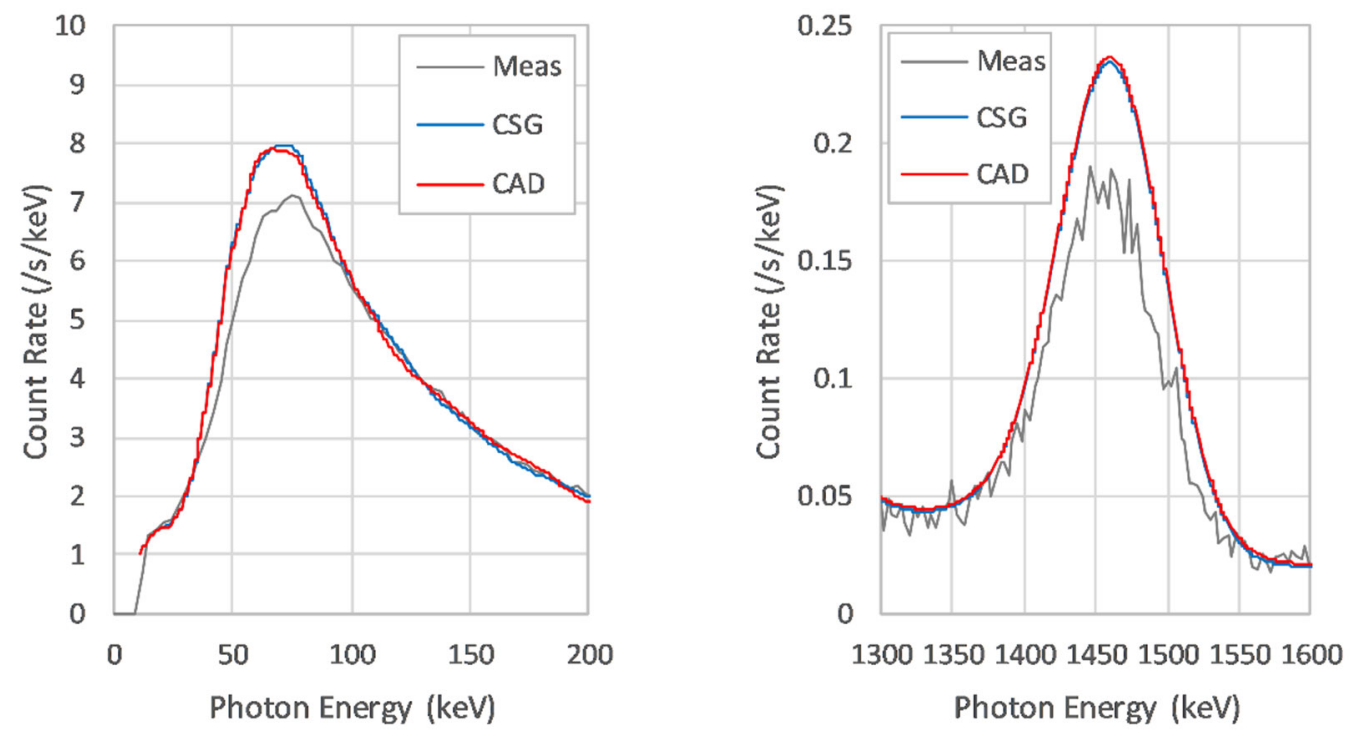

Figure 13. Road 3 background spectra at location $x=10 \mathrm{~m}$ for the low-energy portion of the spectra (left) and near the ${ }^{40} \mathrm{~K}$ peak (right), with measurement data and both CSG and CAD simulations. 


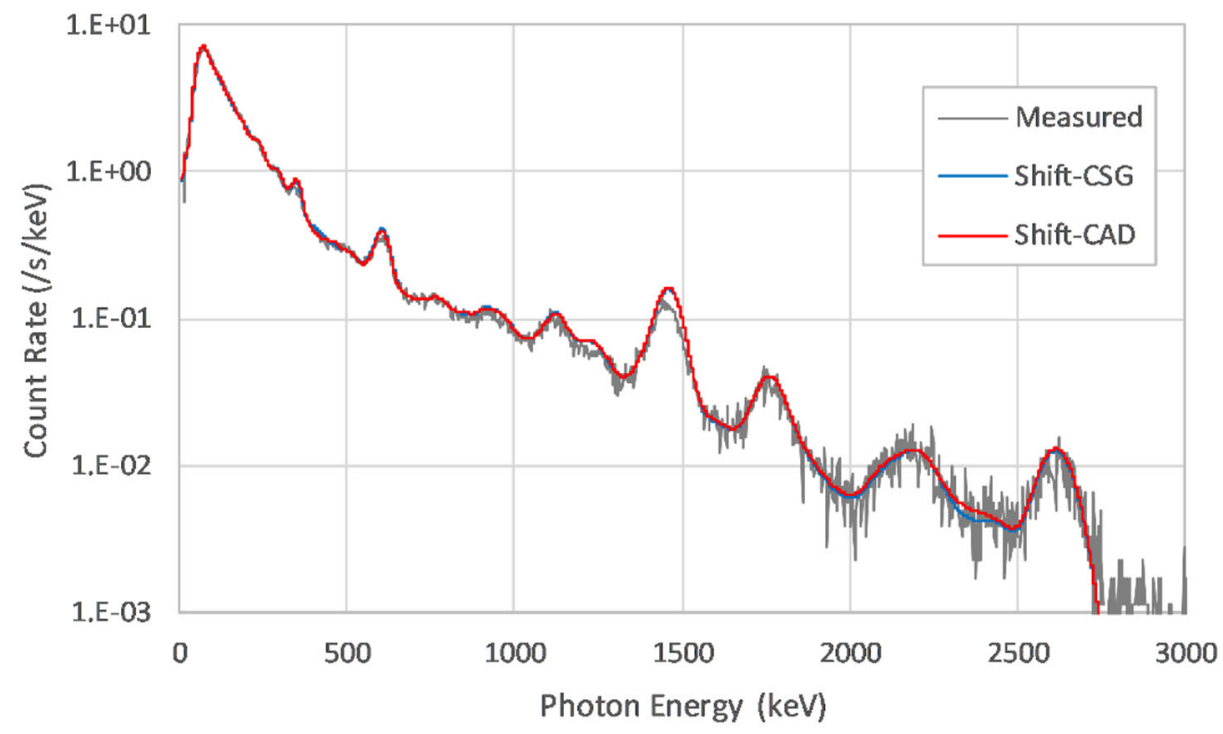

Figure 14. Road 3 background count rate spectra at location $x=40 \mathrm{~m}$ with measurement data and both CSG and CAD simulations. The CSG and CAD simulations agree very well, and both have good agreement with the measured data. Some differences are apparent at the ${ }^{40} \mathrm{~K}$ peak and in the low-energy region.
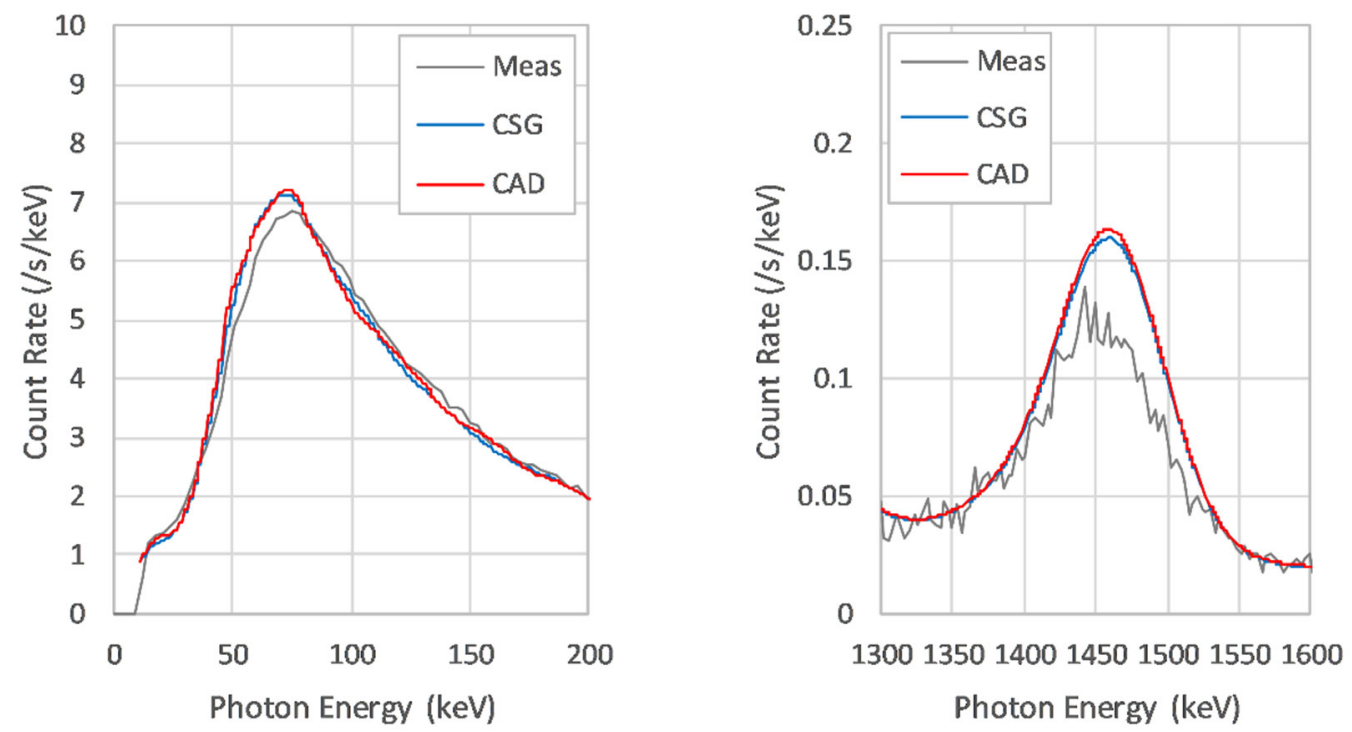

Figure 15. Road 3 background spectra at location $x=40 \mathrm{~m}$ for the low-energy portion of the spectra (left) and near the ${ }^{40} \mathrm{~K}$ peak (right), with measurement data and both CSG and CAD simulations. 


\subsection{ROAD 3 CESIUM SOURCE}

In the 17 measurements for which there was a source, 11 had a clear line of sight between the source and the detector. The signature peak from Cs (the $662 \mathrm{keV}$ photon emitted from ${ }^{137 \mathrm{~m}} \mathrm{Ba}$ ) is very close to the $609 \mathrm{keV}$ peak from ${ }^{214} \mathrm{Bi}$, which is one of the daughters in the decay chain of ${ }^{238} \mathrm{U}$. For each of the four source locations, comparisons of the two model simulations and the measurements are shown for one location. For each comparison, three plots are presented. First, the background-subtracted measurements are compared to the simulations of only the Cs source, from $0-800 \mathrm{keV}$, with uncertainties. The uncertainty for the difference of the two measurements can be very large and is not shown in the plots, although it is readily seen from the large changes in the count rate value in neighboring channels. Second, the region around the Cs peak $(550-750 \mathrm{keV})$ is shown with uncertainties for the measurements and simulations. Third, a comparison of the measurement with the Cs source is compared to the sum of the background and Cs source simulations, also with uncertainties. Source location 1 is shown in Figure 16, source location 2 in Figure 17, source location 3 in Figure 18, and finally, Figure 19 shows source location 4.

\subsection{ROAD A BACKGROUND}

Nine of the background measurements taken along Road A were simulated, starting from Road 3 to the roundabout intersection with Road 2. Most measurements were taken along the centerline of the road, and two were taken near the edge of the road. Measurement location 1 (along Road A, near the curb) and location 6 (on the roundabout, furthest in $+x$ direction) are shown in Figures 20-23. For each location, the entire background spectra are shown for the measurement, along with the CSG simulation and the CAD simulation. Plots near the ${ }^{40} \mathrm{~K}$ line and the low-energy/skyshine region are also shown. Like the Road 3 measurements, uncertainties are not shown in the figures. The uncertainties in the measured data are as follows: $<4 \%$ for $0-500 \mathrm{keV}, 4-7 \%$ for $500-1000 \mathrm{keV}, 5-12 \%$ for $1000-1500 \mathrm{keV}, 7-35 \%$ for 1500 $2000 \mathrm{keV}$, and $18-100 \%$ for above $2000 \mathrm{keV}$. Statistical uncertainties of the Monte Carlo simulations were typically $1-2 \%$ below $2500 \mathrm{keV}$, with some areas having uncertainties up to $10 \%$.

CSG and CAD simulations tended to agree well over the entire energy range. The simulations agreed with the measurements fairly well, with noticeable differences in the low-energy/skyshine region. 

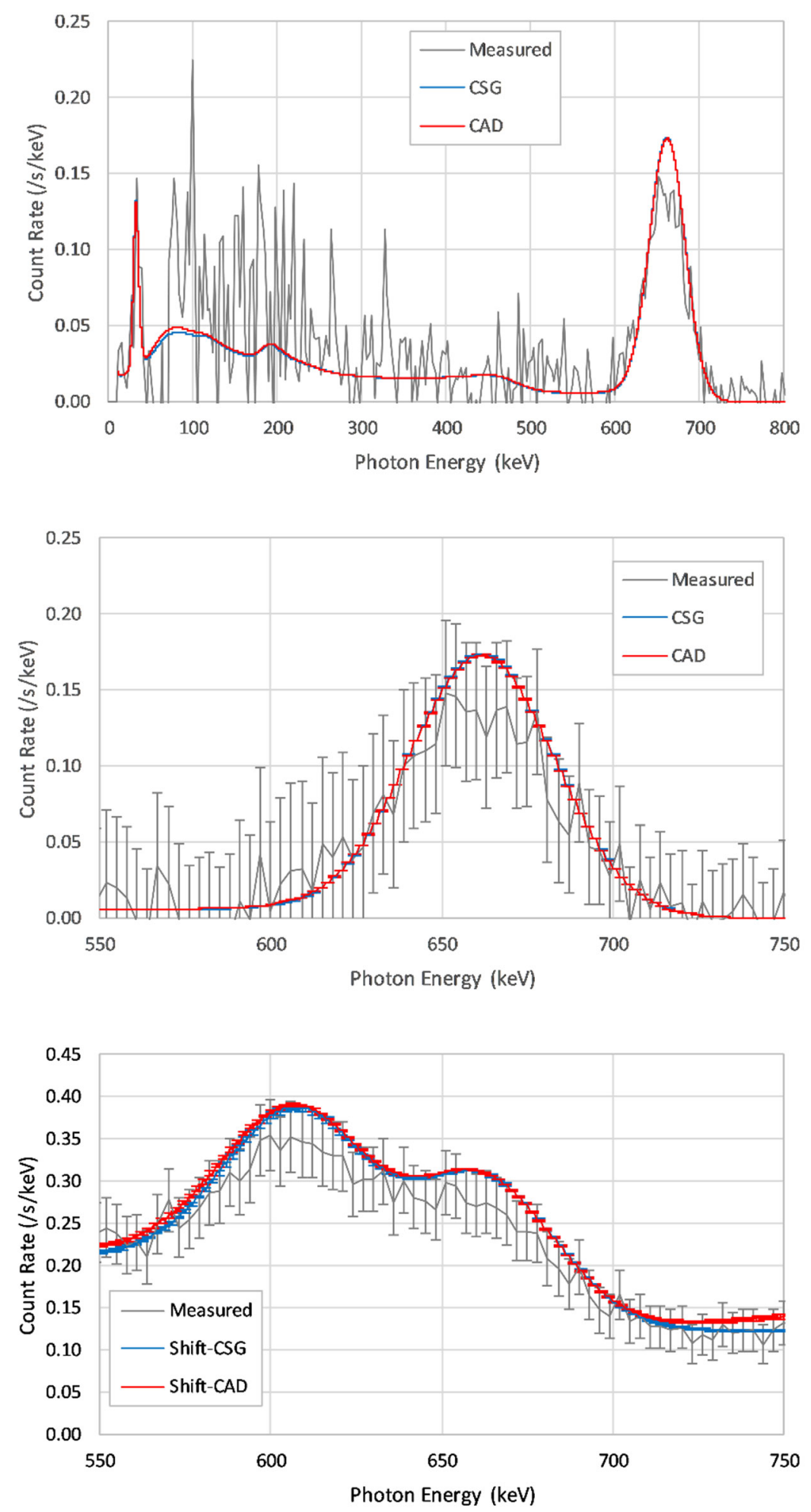

Figure 16. Road 3 spectra at $\mathbf{x}=\mathbf{2 0} \mathbf{m}$ for a source at location 1. Top: Background-subtracted comparison. Middle: Cs peak region of the background-subtracted measurement. Bottom: Cs peak region including the background. 

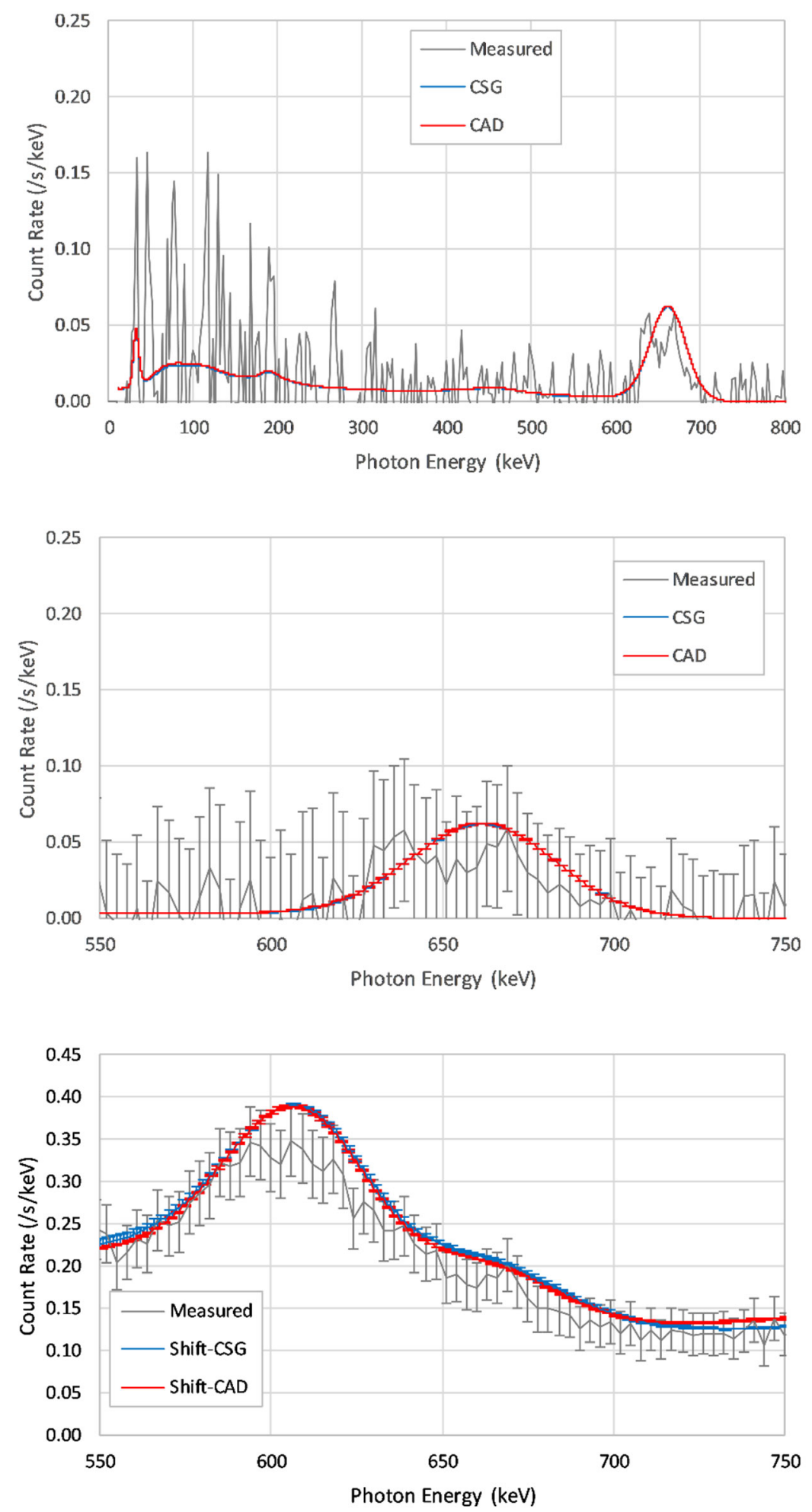

Figure 17. Road 3 spectra at $x=30 \mathrm{~m}$ for a source at location 2. Top: Background-subtracted comparison. Middle: Cs peak region of the background-subtracted measurement. Bottom: Cs peak region including the background. 

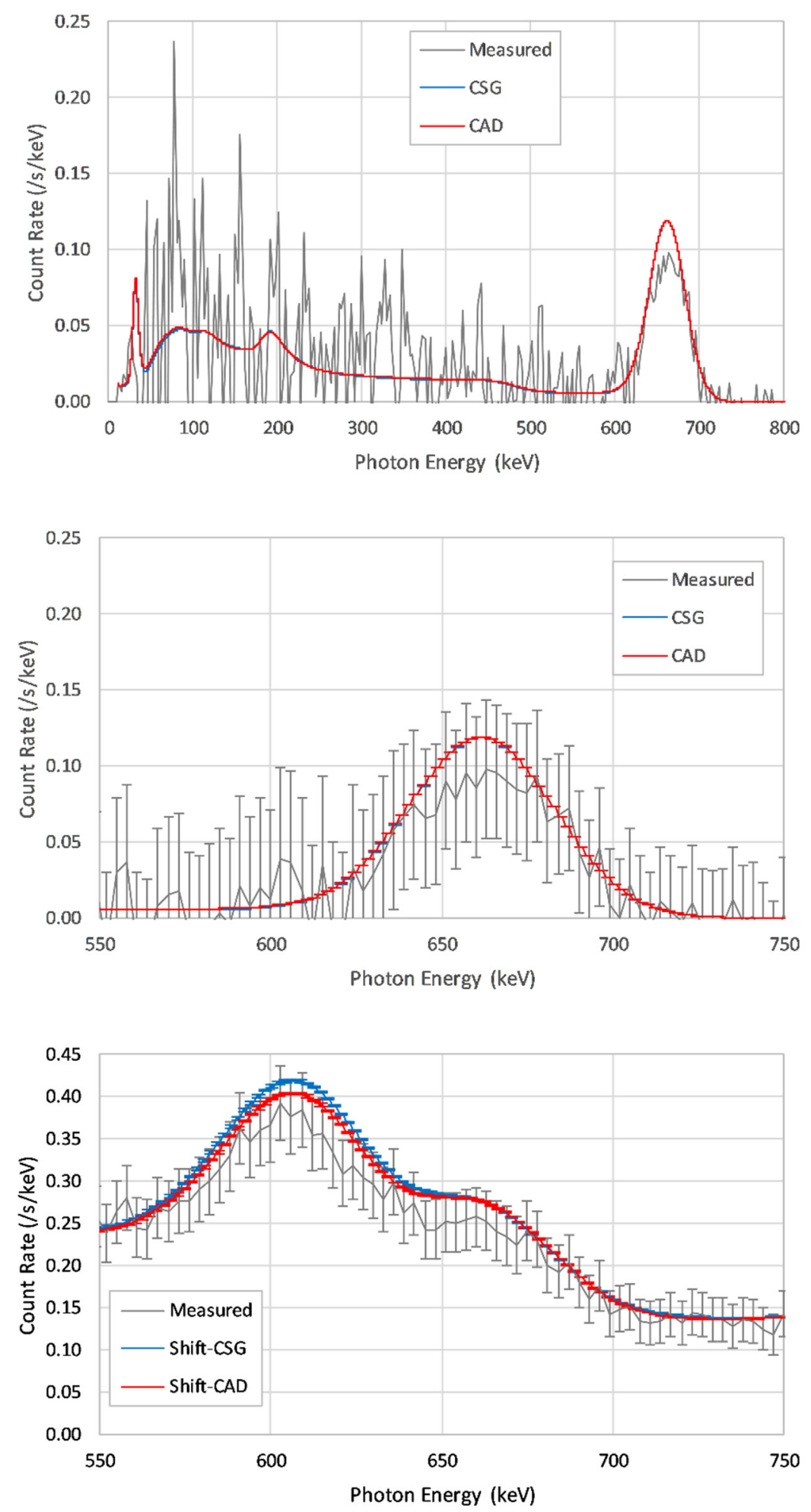

Figure 18. Road 3 spectra at $x=40 \mathrm{~m}$ for a source at location 3. Top: Background-subtracted comparison. Middle: Cs peak region of the background-subtracted measurement. Bottom: Cs peak region including the background. 

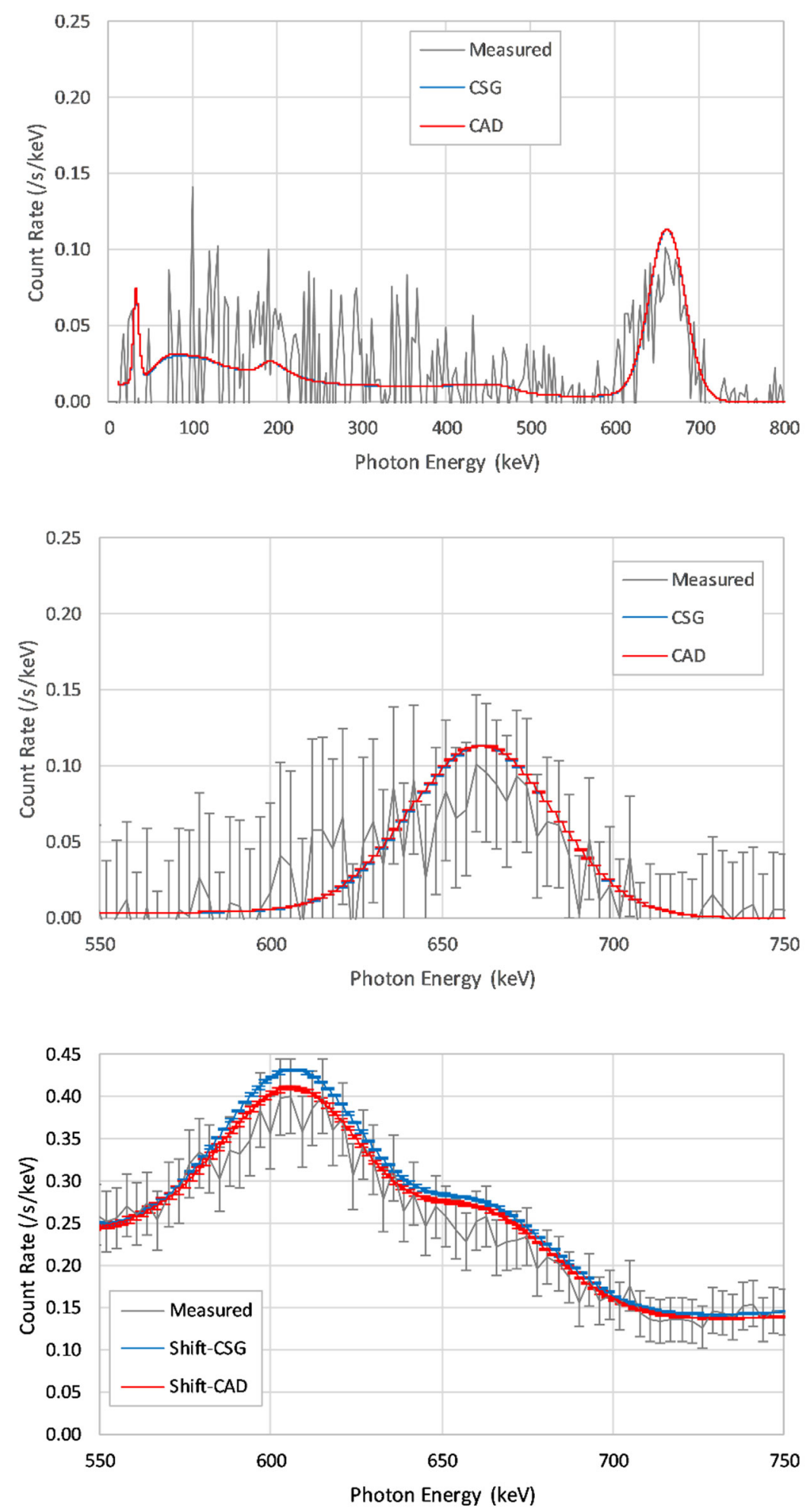

Figure 19. Road 3 spectra at $x=70 \mathrm{~m}$ for a source at location 4 . Top: Background-subtracted comparison. Middle: Cs peak region of the background-subtracted measurement. Bottom: Cs peak region including the background. 


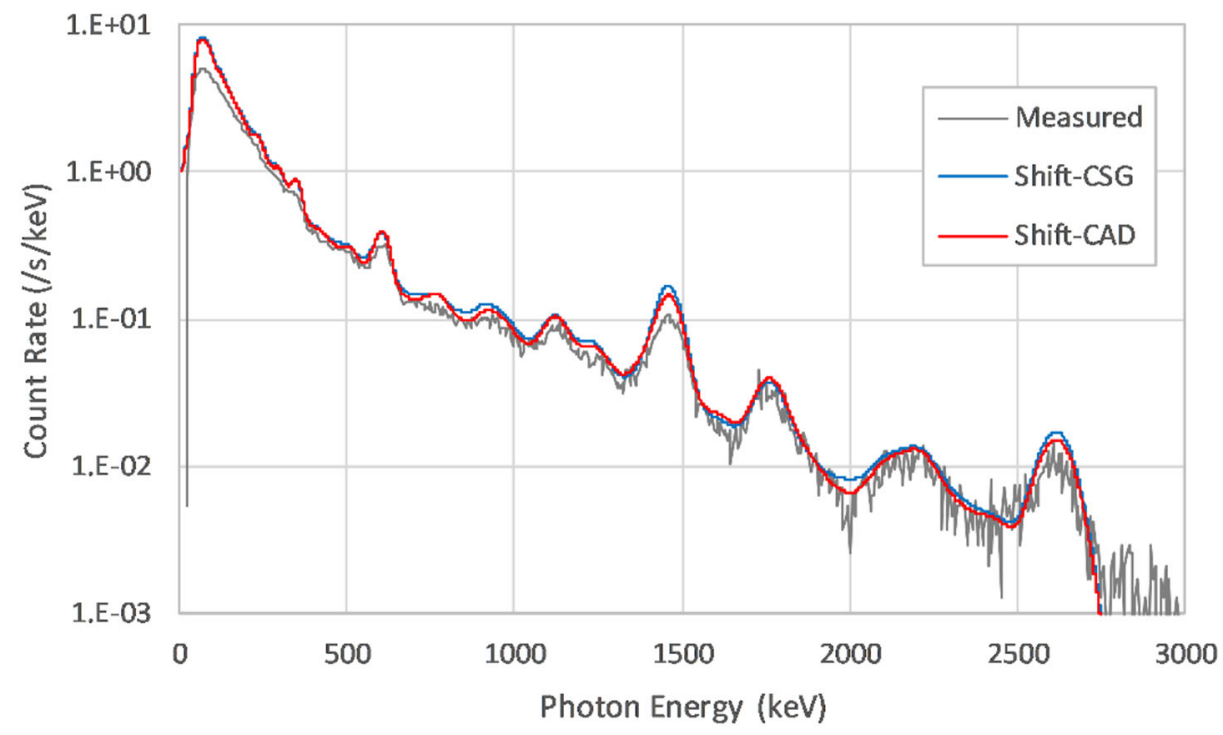

Figure 20. Road A background count rate spectra at location 1 with measurement data and both CSG and CAD simulations. The CSG and CAD simulations agree very well, and both have good agreement with the measured data. Some differences are apparent at the ${ }^{40} \mathrm{~K}$ peak and the low-energy region.
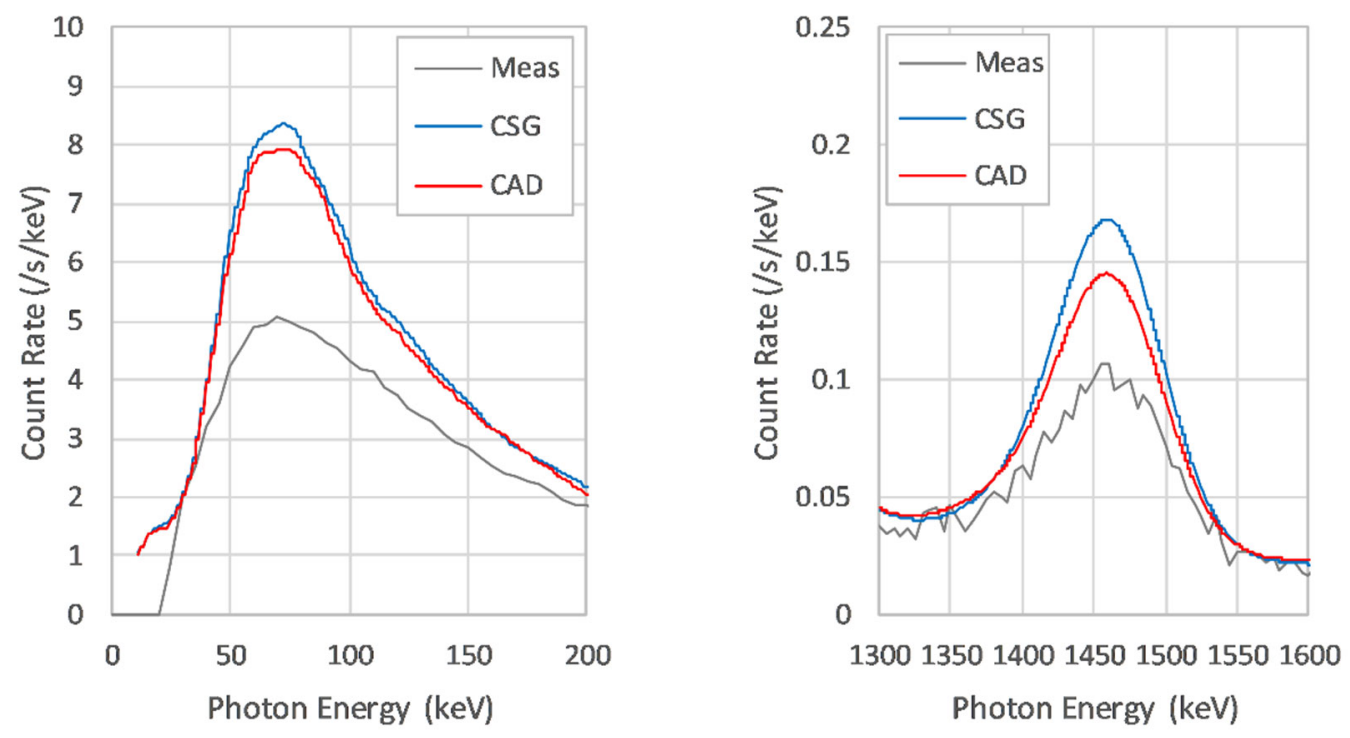

Figure 21. Road A background spectra at location 1 for the low-energy portion of the spectra (left) and near the ${ }^{40} \mathrm{~K}$ peak (right), with measurement data and both CSG and CAD simulations. 


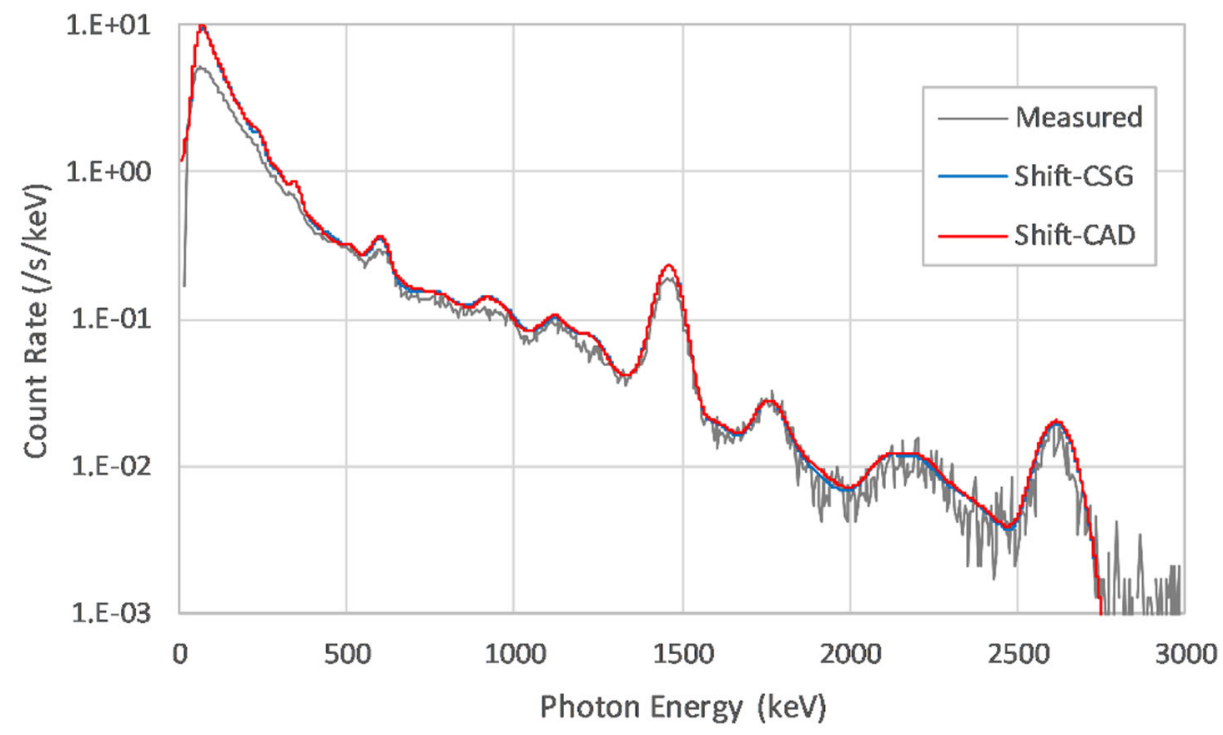

Figure 22. Road A background count rate spectra at location 6 with measurement data and both CSG and CAD simulations. The CSG and CAD simulations agree very well, and both have good agreement with the measured data. Some differences are apparent at the ${ }^{40} \mathrm{~K}$ peak and the low-energy region.
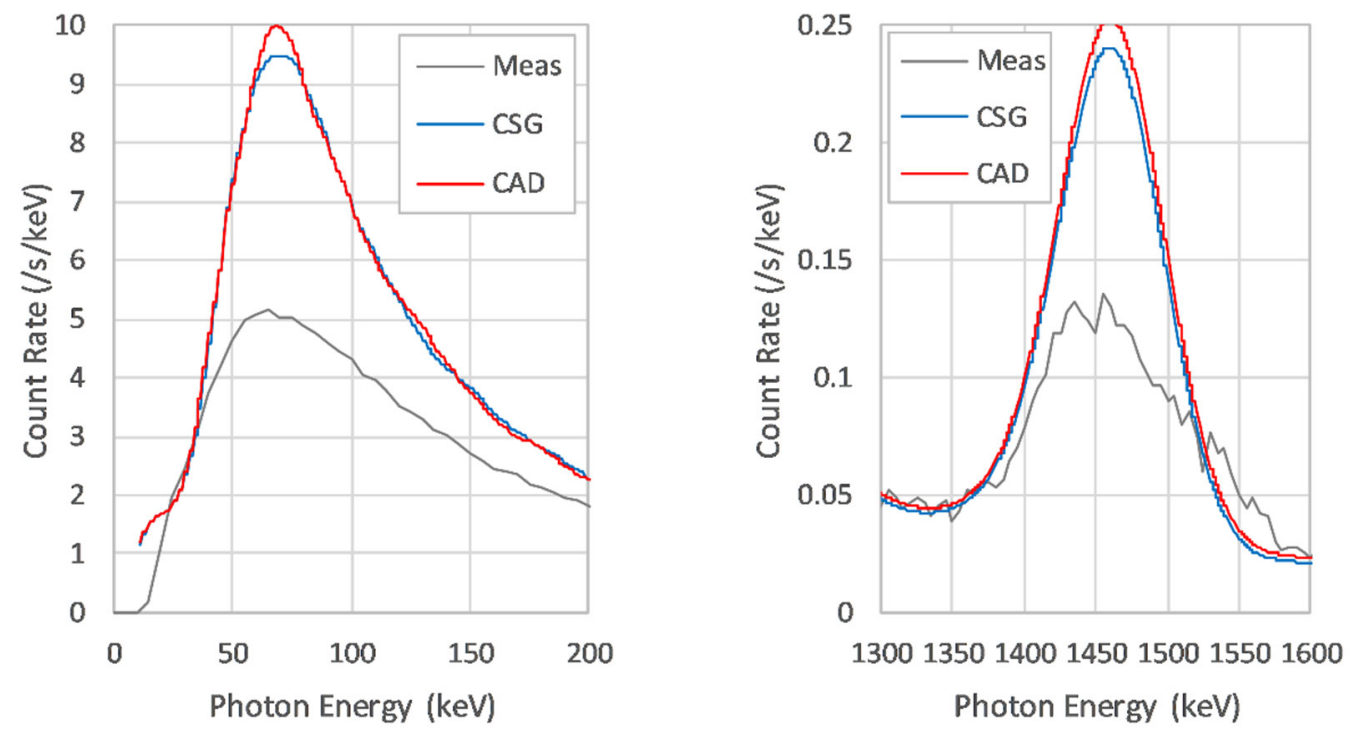

Figure 23. Road A background spectra at location 6 for the low-energy portion of the spectra (left) and near the ${ }^{40} \mathrm{~K}$ peak (right), with measurement data and both CSG and CAD simulations. 


\section{COMPARISON OF CAD AND CSG}

As seen in Figures 12-35, the spectral results from the simulations are in very good agreement, even though the CSG model has more details (curbs and sidewalks, windows and doors in the buildings, rubble zones with some buildings, and gravel layers beneath the asphalt and concrete roadways). The agreement between the two calculations is much closer than the agreement of either with the actual measurements. For simulating the measurements made at the FTIG site, the greater level of detail in the CSG model may not have been necessary. Future studies could examine how much detail is needed in the geometry model (CSG or CAD) to accurately simulate urban search measurements.

Regarding calculation speed, it was expected that raytracing on the CAD geometry would have been slower that the CSG geometry in terms of the number of particle histories per minute. As shown in Table 2, this was not the case. The Monte Carlo calculations using the CAD geometry processed more histories than when using the CSG geometry except in the Road 3 background simulation. This is most likely because the CSG model contains more detail than the CAD model.

\section{Table 2. Particle processing rate for the twelve simulations (millions of particles per minute)}

\begin{tabular}{cl|cc}
\hline \multirow{2}{*}{ Simulation } & \multicolumn{2}{c}{ Geometry } \\
\cline { 3 - 4 } & Background & 6.0 & 4.5 \\
\hline \multirow{2}{*}{ Road 3 } & Source 1 & 2.6 & 4.4 \\
& Source 2 & 1.7 & 4.2 \\
& Source 3 & 3.8 & 5.2 \\
& Source 4 & 2.2 & 4.7 \\
\hline Road A & Background & 7.6 & 4.0 \\
\hline
\end{tabular}




\section{SUMMARY}

Predictions of the $\mathrm{NaI}$ detector response were made with the Shift code for both a threat source and background using first principles: measured concentrations of the NORM components, full 3D transport in a detailed model, and a detector response function derived from a series of basic transport calculations. Only the energy resolution parameters of the detectors were chosen to match the detectors used in the measurements. Simulations using a traditional hand built CSG geometry and a CAD geometry derived from LiDAR measurements gave very similar responses. Simulations using both geometries matched the measurements well: even the low-energy peak due to skyshine was reasonable given the assumptions of a perfectly flat area around the main FTIG site with uniform NORM concentration values. Simulations did require long times on a multiprocessor computer to converge the energy-dependent flux tallies to low uncertainties at each detector location.

With a validated virtual testbed, detailed simulations could be used to predict detector responses for a variety of scenarios. Response functions for different detectors could be used with the same flux data, without rerunning Shift, to predict those responses. Simulated detector responses would be useful for planning measurement campaigns and exercises. Simulated data could be used to evaluate and compare detection/identification algorithms. Unlike using actual measured data for algorithm testing, when using Monte Carlo simulation of background and source measurements with CSG and CAD geometries, everything in the simulations is known, so there is no question as to how the algorithm is supposed to respond. Different algorithms could be tested against the same set of synthetic data, giving a true comparison since it was the exact same situation for each algorithm. Simulation could also be used to understand the impacts of clutter in between the detector and the threat source or simply to explore what fraction of the count rate spectrum is coming from different sources-background or scattering.

\section{ACKNOWLEDGMENTS}

This work was sponsored by the Enabling Capabilities for Nonproliferation and Arms Control Program Area of the Office of Defense Nuclear Nonproliferation Research and Development, National Nuclear Security Administration. The authors would like to thank M. S. Lance K. McLean, Alexander A. Plionis, and the team of technicians from RSL who collected the Road 3 background and source measurements at the FTIG site. The authors also thank ORNL reviewer Eric D. Sword and Mathew W. Swinney for their helpful suggestions. Some of this research used resources of the National Energy Research Scientific Computing Center (NERSC), a US Department of Energy Office of Science User Facility operated under Contract No. DE-AC02-05CH11231. 


\section{REFERENCES}

1. A. D. Nicholson, I. Garishvili, D. E. Peplow, D. E. Archer, W. R. Ray, M. W. Swinney, M. J. Willis, G. G. Davidson, S. L. Cleveland, B. W. Patton, D. E. Hornback, J. J. Peltz, M. S. L. McLean, A. A. Plionis, B. J. Quiter, and M. S. Bandstra, "Multi-Agency Urban Search Experiment Detector and Algorithm Test Bed," IEEE Transactions on Nuclear Science 64, 7, 1689-1695 (2017); DOI: 10.1109/TNS.2017.2677092.

2. D. E. Archer, M. S. Bandstra, D. S. Bolme, S. L. Cleveland, J. C. Curtis, G. G. Davidson, I. Garishvili, J. O. Johnson, A. K. Mikkilineni, M. S. L. McLean, A. D. Nicholson, D. E. Peplow, A. A. Plionis, M. J. Poska, B. J. Quiter, W. R. Ray, A. J. Row, I. R. Stewart, M. W. Swinney, M. J. Willis, Mid-Project Report for the Modeling Urban Scenarios and Experiments (MUSE) Project, ORNL/TM-2018/964, Oak Ridge National Laboratory, Oak Ridge, Tennessee, August 2018.

3. G. A. Sandness, J. E. Schweppe, W. K. Hensley, J. D. Borgardt, and A. L. Mitchell, "Accurate Modeling of the Terrestrial Gamma-Ray Background for Homeland Security Applications,” 2009 IEEE Nuclear Science Symposium Conference Record (NSS/MIC), 24 Oct. -1 Nov. 2009; DOI: 10.1109/NSSMIC.2009.5401843.

4. United States Geological Survey, "Terrestrial Radioactivity and Gamma Ray Exposure in the United States and Canada," Open File Report 2005-1413, pubs.usgs.gov/of/2005/1413/.

5. R. T. Kouzes, J. H. Ely, B. D. Geelhood, R. R. Hansen, E. A. Lepel, J. E. Schweppe, E. R. Siciliano, D. J. Strom, and R. A. Warner, "Naturally Occurring Radioactive Materials and Medical Isotopes at Border Crossings," 2003 IEEE Nuclear Science Symposium Conference Record, 19-25 October 2003, 2, 1448-1452; DOI: 10.1109/NSSMIC.2003.1351967.

6. M. Cooper, "Naturally Occurring Radioactive Materials (NORM) in Australian Industries - Review of Current Inventories and Future Generation, ERS-006," a report prepared for the Radiation, Health and Safety Advisory Council (2005).

7. IAEA, "Extent of Environmental Contamination by Naturally Occurring Radioactive Material (NORM) and Technological Options for Mitigation," Tech Report Series No. 419 (December 2003).

8. Fort Indiantown Gap National Guard website, www.ftig.ng.mil/Training/Urban-and-CollectiveTraining-Sites/Combined-Arms-Collective-Training-Facility (accessed January 2020).

9. A. D. Nicholson, D. E. Peplow, M. J. Willis, and D. E. Archer, "Generation of Synthetic Data for a Radiation Detection Algorithm Competition," accepted for IEEE Transactions on Nuclear Science 67, 8, 1968-1975, (2020); DOI: 10.1109/TNS.2020.3001754.

10. M. W. Swinney, D. E. Peplow, B. W. Patton, A. D. Nicholson, D. E. Archer, and M. J. Willis, “A Methodology for Determining the Concentration of Naturally Occurring Radioactive Materials in an Urban Environment," Nuclear Technology, 203, 3, 325-335 (2018); DOI: 10.1080/00295450.2018.1458558.

11. M. S. Bandstra, T. J. Aucott, E. Brubaker, D. H. Chivers, R. J. Cooper, J. C. Curtis, J. R. Davis, T. H. Joshi, J. Kua, R. Meyer, V. Negut, M. Quinlan, B. J. Quiter, S. Srinivasan, A. Zakhor, R. Zhang, and K. Vetter, "RadMAP: The Radiological Multi-sensor Analysis Platform," Nuclear Instruments and Methods in Physics Research Section A: Accelerators, Spectrometers, Detectors and Associated Equipment, 840, 59-68 (2016). DOI: 10.1016/j.nima.2016.09.040

12. M. S. Bandstra, B. J. Quiter, J. C. Curtis, K. J. Bilton, T. H. Y. Joshi, R. Meyer, V. Negut, K. Vetter, D. E. Archer, D. E. Hornback, D. E. Peplow, C. E. Romano, M. W. Swinney, T. L. McCullough, and M. S. L. McLean, "Attribution of Gamma-Ray Background Collected by a Mobile Detector System to Its Surroundings Using Panoramic Video," Nuclear Instruments and Methods in Physics Research 
Section A: Accelerators, Spectrometers, Detectors and Associated Equipment, 954 (2020) 161126. DOI: 10.1016/j.nima.2018.08.085

13. M. Salathe, M. S. Bandstra, B. J. Quiter, J. C. Curtis, "Using 3D-Scene Data from a Mobile Detector System to Model Gamma-Ray Backgrounds," 2019 IEEE Nuclear Science Symposium and Medical Imaging Conference Record, April 2020. DOI: 10.1109/NSS/MIC42101.2019.9059835

14. T. M. Pandya, S. R. Johnson, T. M. Evans, G. G. Davidson, S. P. Hamilton, and A. T. Godfrey, "Capabilities, Implementation, and Benchmarking of Shift, a Massively Parallel Monte Carlo Radiation Transport Code," Journal of Computational Physics, 308, 239-272 (2016); DOI: 10.1016/j.jcp.2015.12.037.

15. D. E. Peplow, K. Banerjee, G. G. Davidson, I. R. Stewart, M. W. Swinney, and J. N. Wagner, "Preliminary Validation of the Shift Monte Carlo Code for Fixed-Source Radiation Transport Problems," Nuclear Technology, 206, 1, 107-125 (2020); DOI: 10.1080/ 00295450.2019.1625663.

16. T. M. Evans, A. S. Stafford, R. N. Slaybaugh, and K. T. Clarno, "Denovo: A New ThreeDimensional Parallel Discrete Ordinates Code In SCALE," Nuclear Technology, 171, 2, 171-200 (2010); DOI: 10.13182/NT171-171.

17. S. P. Hamilton, T. M. Evans, S. R. Slattery, "Continuous-energy Monte Carlo Neutron Transport on GPUs in Shift," Transactions of the American Nuclear Society, 118, 401-403 (2018).

18. J. C. Wagner, S. W. Mosher, T. M. Evans, D. E. Peplow, and J. A. Turner, "Hybrid and Parallel Domain-Decomposition Methods Development to Enable Monte Carlo for Reactor Analyses," Progress in Nuclear Technology, 2, 815-820 (2011); DOI: 10.15669/pnst.2.815.

19. R. S. Baker and K. R. Koch, "An $\mathrm{S}_{\mathrm{N}}$ Algorithm for the Massively Parallel CM-200 Computer," Nuclear Science and Engineering, 128, 3, 312-320 (1998); DOI: 10.13182/NSE98-1.

20. G. G. Davidson, T. M. Evans, J. J. Jarrell, S. P. Hamilton, T. M. Pandya, and R. N. Slaybaugh, "Massively Parallel, Three-Dimensional Transport Solutions for the $k$-Eigenvalue Problem," Nuclear Science and Engineering, 177, 2, 111-125 (2014); DOI: 10.13182/NSE12-101.

21. J. C. Wagner and A. Haghighat, "Automated Variance Reduction of Monte Carlo Shielding Calculations using the Discrete Ordinates Adjoint Function," Nuclear Science and Engineering, 128, 2, 186-208 (1998); DOI: 10.13182/NSE98-2.

22. J. C. Wagner, D. E. Peplow, and S. W. Mosher, "FW-CADIS Method for Global and Semi-Global Variance Reduction of Monte Carlo Radiation Transport Calculations," Nuclear Science and Engineering, 176, 1, 37-57 (2014); DOI: 10.13182/NSE12-33.

23. B. T. Rearden and M. A. Jessee, Eds., "SCALE Code System," ORNL/TM-2005/39 Version 6.2.3, Oak Ridge National Laboratory (March 2018). Available from Radiation Safety Information Computational Center as CCC-834; DOI: 10.2172/1426571.

24. T. Goorley, M. James, T. Booth, F. Brown, J. Bull, L. J. Cox, J. Durkee, J. Elson, M. Fensin, R. A. Forster, J. Hendricks, H. G. Hughes, R. Johns, B. Kiedrowski, R. Martz, S. Mashnik, G. McKinney, D. Pelowitz, R. Prael, J. Sweezy, L. Waters, T. Wilcox, and T. Zukaitis, "Initial MCNP6 Release Overview," Nuclear Technology, 180, 3, 298-315 (2012); DOI: 10.13182/NT11-135.

25. S. Stimpson, T. Pandya, K. Royston, and B. S. Collins, "Impact of Radial Reflector Fidelity on Neutronics and Vessel Fluence Simulations," Nuclear Technology (2020); DOI: 10.1080/00295450.2020.1770557.

26. T. J. Tautges, P. P. H. Wilson, et al, "Acceleration Techniques for Direct Use of CAD-Based Geometries in Monte Carlo Radiation Transport," Proc. of the 2009 Int'l Conf. on Mathematics, Computational Methods \& Reactor Physics, Saratoga Springs, NY, May 3-7 (2009). 
27. D. E. Peplow, M. W. Swinney, G. G. Davidson, A. D. Nicholson and B. W. Patton, "Initial Modeling of Urban Search Measurements," Transactions of the American Nuclear Society, 116, 913-916 (2017).

28. W. Hess, D. Kohler, H. Rapp, and D. Andor, "Real-Time Loop Closure in 2D LIDAR SLAM," in 2016 IEEE International Conference on Robotics and Automation (ICRA), 1271-1278, Stockholm, Sweden, May 16-21, 2016.

29. Python Software Foundation, "Python Language Reference, Version 2.7," http://www.python.org accessed 08/04/2020.

30. M. Skroch, S. J. Owen, M. L. Staten, R. W. Quadros, B. Hanks, B. Clark, T. Hensley, R J. Meyers, C. Ernst, R. Morris, C. McBride and C. Stimpson, The CUBIT Geometry and Mesh Generation Toolkit 15.4 User Documentation, SAND2019-3478 W, Sandia National Laboratories, https://cubit.sandia.gov, accessed 08/05/2020.

31. "DAGMC: Direct Accelerated Geometry Monte Carlo Toolkit," University of Wisconsin-Madison, https://svalinn.github.io/DAGMC, accessed 10/17/2019.

32. B. M. Smith, "Robust Tracking and Advanced Geometry for Monte Carlo Radiation Transport," Ph.D. dissertation, University of Wisconsin-Madison, 2011.

33. T. J. Tautges, C. Ernst, C. Stimpson, R. J. Meyers, and K. Merkley, MOAB: A Mesh Oriented Database, SAND2004-1592, Argonne National Laboratory, Argonne, IL, 2004.

34. Argonne National Laboratory SIGMA Project, "MOAB: A Mesh Oriented Database," https://sigma.mcs.anl.gov/moab-library, accessed 08/04/2020.

35. R. J. McConn, C. J. Gesh, R. T. Pagh, R. A. Rucker, and R. G. Williams III, "Compendium of Material Composition Data for Radiation Transport Modeling," PIET-43741-TM-963, PNNL-15870, Rev. 1, Pacific Northwest National Laboratory (March 2011); DOI: 10.2172/1023125.

36. C. Celik, D. E. Peplow, M. W. Swinney, and G. G. Davidson, "A Directional Detector Response Function for Anisotropic Detectors," Nuclear Science and Engineering, 193, 12, 1355-1370 (2019); DOI: $10.1080 / 00295639.2019 .1631028$. 